\title{
Implementation model of a universe based on information
}

\author{
Alessandro Capurso \\ Nov. 30, 2021 - Turin, Italy \\ This work is given as a first draft of a framework better clarified in the following contributions: \\ "The potential of a thick present through undefined causality and non-locality" (doi.org/10.31219/osf.io/52xyd) \\ and "A simple holographic universe" (doi.org/10.31219/osf.io/pa86f)
}

\begin{abstract}
Starting from an Information and Communication Technologies perspective focused on implementability, we propose a model of the universe based on a memory network. Spacetime is described through finite and discrete information of non-local spatial correlations elaborated on a discrete processing cycle (as a thick present). The need for logical consistency in the information is connected to a relativity in respect to global scales of reference in an interconnected universe. The quantized correlation potential (from which space emerges as imaginary in each cycle) is summarized in a fundamental causal memory with a log-perspective through a Dual-Tree Complex Wavelet Transform and persists along the evolution cycles as closed time-like curves (CTC) within the thick present considered. CTC, described as memory-loops, are introduced to assure logical consistency of the information potential. We then propose an interpretation of elementary particles connected to a holographic description, extended in the model to $E R=E P R=C T C$. Finally, we identify a physical implementation of the model in black holes, clarifying how fundamental constants could be defined in the spacetime patch emerging beyond the singularity and proposing quantum fields as wavelets along the radius. Additional elaborations on both metric and space expansion in the proposed model are provided in the dedicated Annexes.
\end{abstract}

Keywords: information; entanglement; logical consistency; memory; network; thick present; quantum gravity; holography; CTC; black hole;

\section{Introduction}

Many research paths towards a theory of Quantum Gravity (QG), able to describe spacetime and the universe evolution in a quantum formalism and give a more general framework to the current theories, start from the concept of information, as a lens through which both quantum mechanics (QM) and spacetime could be interpreted. This paper follows a similar path, approaching a quantum description of phenomena from an Information and Communication Technologies (ICT) perspective and focusing on the requirements for a physically implementable system.

It is worth investigating the description of a toy universe in which spacetime, in all its content of mass and energy, instead of being just a "stage" or background for QM, could emerge from information, with observables in line with QM. Starting with a simple model and understanding which engineering hypotheses are needed to build a full evolving spacetime from information, could give us insights on how our universe, when described through the lens of information, could behave.

From now on, we will refer to the proposed model as Fantasia, a tribute to the universe where every story could exist.

The reader should be warned that the concept of "spacetime from information" and the reasoning on possible algorithms able to create Fantasia spacetime from information are not promoting the idea that "we live in a simulation" (which is an unneeded speculation). To overcome this temptation, a possible physical realization of the proposed model in the current universe will be considered in the last part of this paper.

\footnotetext{
\# ale.capurso@gmail.com - orcid.org/0000-0003-3277-3588
} 


\section{Engineering hypotheses and design}

\section{Spacetime in the context of information}

The concept of "observer" is key in a QM description of phenomena, but its meaning has always been open to several interpretation. In a context of "it from bit", questioning if the underlying information and the emerging spacetime are independent from the observers could be meaningless: there are no external observers or isolated labs in a universe without a "spacetime stage", as all observers are part of the information from which the observed phenomena emerge. Observers could make local measurements, relative to their point of view on spacetime as Relativity indicates, but the concept of relativity itself seems to require the emerging spacetime to be interconnected at a more fundamental level. Actually, to be relative, any information cannot be isolated or external, at least in respect to a global perspective, as it is in the confrontation with a global perspective that all single local ones find their relative coherence and the global one finds its consistency. Eventually, it could be in the wrong hypothesis of isolated systems or labs that many theories on spacetime find hard to "go quantum" and a local and a global coherence might be lost.

Given these considerations, the fundamental information (as well as observables) must be logically consistent, with no logical contradiction between a local and relative point of view and a global one. Logical consistency requires that information should not be under or over determined and it is a weaker requirement than causality (as debated in [1]).

Beyond requiring logical consistency on information, to have a physically realizable universe, we need as well to upper bound the information required to describe it. Actually, in the context of "spacetime from information", no implementable or existing universes should need infinite or unbounded information to be physically representable. From a mathematical perspective, a finite information cannot describe the Real continuum (as also debated in [2]), hence the spacetime fabric in Fantasia is considered discrete, and its physical observables must be discrete and relative to global units of reference.

In a discrete spacetime, it seems logic to consider the passage of time as well as occurring in discrete steps, below which it is meaningless to define the concept of time. Towards a discrete passage of time and a discrete space, also mass should be considered from a perspective relative to the scale of absolute references. The relevance of the Planck mass in the evaluation of the quantum nature of spacetime has also been recently highlighted in [3] and [4]. We could then consider Planck units as universal references (both in Fantasia as in our universe). Giving Planck length $L$, Planck mass $M$, Planck time $T$ and considering $F=1 / T$ as the ideal maximum degree of freedom in $f$ (angular frequency or just frequency for short), we can express the fundamental constants (from which they are derived) as following:

$$
c=\frac{L}{T}=L F ; \quad \hbar=\frac{M L^{2}}{T}=M L^{2} F ; \quad G=\frac{L}{M} c^{2}
$$

Finally, as no natural phenomena should be imagined without a sense of parsimony, it could be wise to minimize the information needed to describe Fantasia. As in a universe based on information what should matter is the current global perspective on information (in which and from which all relative local perspectives could be accounted or derived), no precise reconstruction of spacetime at a given far past instant should be required. Information of the far past states is not needed beyond the current state dependencies (as a causal relation of the current state with the far past ones) and what happened in the past states cannot be changed. Only a summary of the causal past dependencies is needed, further limiting the growth of required information.

On the same line, we can consider that no information about the future cycles should be needed for a logically consistent emergence of spacetime, as the future of Fantasia is intended as still undefined and open. Further investigations on the concept of time and of evolution will be left to the coming chapter. 


\section{Perspectives on the idea of Time}

We started picturing a universe in which spacetime could emerge from information and elaborated on possible engineering hypotheses needed to implement it. We have required logical consistency, upper bounded the information (deducing a discrete spacetime) and tried to minimize it. Giving that the understanding of time is at the root of QG theories, it is worth listing the multiple perspectives on the topic, to figure out if the hypotheses considered so far are consistent and if it is possible to get further insights.

The interpretation of the nature of time has seen two opposite descriptions. The first, called Eternalism, is often related to the theory of Relativity and consider an ever-existing time, real in both the past and the future, and of which we perceive only limited cones of information. Even in Relativity we could always identify a possible "global perspective" (coherent with the local observations) considering as a preferred reference frame on spacetime the point of view at infinity. The opposite perspective, known as Presentism in physical theories, is connected to the idea of evolution itself and pictures time as everchanging: a vanishing present compressed between past and future instants. The focus is on the difference between the past, already happened and irreversible, and the future, still undefined and open. The axis of time seems to become real only in the current instant and in the causal consequences of the past ones.

The interpretations of QM, in an ironic and iconic uncertainty, stand on both sides of the diatribe. There are timeless formulations (where the variable $t$ disappears in the equations), resulting in an "eternal picture" of what is possible, as well as interpretations that focus on evolution and on the irreversibility of events, closer to Presentism.

Considering a Presentism approach, there are several theories that consider evolution in discrete cycles. Finite-State Classical Mechanics (proposed in [5]) is based on Lattice Dynamics, where the information is described through discrete positions and evolution rules are based on discrete cycles (referred as cellular automata models). Signal-State Quantum Mechanics, further developed in a theory of Quantized Detector Networks (presented in [6]) describes particles as storing entangled phase information and focus on observers and their apparatus, as a realization of Heisenberg's "instrumentalist approach" to quantum physics.

Physical laws in a classical description are time symmetric and there are several QM symmetric approaches too, even if the collapse of the wave function poses limits to a longterm symmetry. From a computational perspective on information, recent "gargantuan simulations" (reported in [7]) showed that time seems irreversible at the most fundamental level, beyond thermodynamic reasons. A simple 3-bodies system "would require an accuracy of smaller than the Planck length in order to produce a time-reversible solution": what had been already can influence the present but cannot be changed and, beyond fundamental physical limits, cannot even be known with certainty.

Even in a context of irreversibility of events, we could still consider a QM time symmetric approach. The idea of an emerging causal reality connected to the superposition of both a forward and a backward propagating wave was proposed in the Two-State-Vector Formalism (TSVF), introduced in [8] and further elaborated recently in a more extensive theory, including elements of Energetic Causal Sets (ECS), in [9] and [10]. Indeterminism in time order has also been considered as a source of quantum information. Possible quantum temporal superposition has been investigated in [11], where the analysis of temporal order in a quantum framework together with Bell's inequalities lead to the identification of possible "causal inequalities".

Beside physics, the common experience sees a forward and causal progression, associated in physical interpretations to thermodynamics and defined on average or macroscopically, as phenomena naturally tend to higher entropy states. Neuroscience studies picture a perception of time in our brain connected to a "perceived present". Our neurons equalize different asynchronous stimuli and derive a coherent picture of the surrounding space in each perceived instant. Our perception is not always accurate but to our brain looks coherent and this is enough for a perception of a "flowing time": a past causally connected to the future through what is happening in the perceived present. 
Taking into account all the mentioned perspectives on time, we could consider the concept of a "thick present" (also elaborated in [12] following an intuitionist mathematical language) as the elementary quanta in the passage of time. We could consider a minimum time-delta $T$ like a $\pi$ rotation, as to see things from a different perspective and as if $2 T$ (or equivalently $2 \pi$ ) were needed for a full cycle. We could consider these discrete $2 T$ steps, whit $T$ equal to the Plank time, as the elaboration cycle of the information from which the spacetime of Fantasia emerges.

A thick present could account for superposition of perspectives from a near past and a near future, resulting time symmetric within its thickness and assuring logical consistency between what it was and what it could be in the current cycle. Information could be kept as a potential in case no events occurred in the elaboration cycle, while events of collapse or "measurements" would be interpreted as irreversible, also in line with the computational perspective. From logical consistency and from the irreversibility of events, a thermodynamic arrow of time (in line with causality orientation) could also be considered as emerging (in the succession of the thick present instants) in the memory of the observers. In this context, as Freeman Dyson wrote in his "Thought Experiments in Honor of John Archibald Wheeler", the only role of the observer becomes the distinction between a classical past and a still open and probabilistic future. Eventually, the thick present could identify a simple, bounded and minimized information to describe Fantasia and could be related to the $2 T$ elaboration cycle as well as to the information needed for the emergence of spacetime in each cycle.

\section{Evolving memories of imaginary distances}

We have considered a universe emerging from information and elaborated on the possible requirements from an implementability perspective. We have required logical consistency and the use of discrete global units of reference for a possible coherent absolute perspective on information, but we still have to define the information we would need to generate its spacetime. We could consider as elementary information the general idea of "connection among spacetime points", intended as a link of correlation and entanglement among distant elements in a global network. The more correlation links are available in a region of spacetime, the more information we will locally have. From an ICT perspective, a simple model of correlation could rely on the sharing of a common phase or code in respect to a common external reference, like a "shared secret". In the simplest scenario, the external reference could be unique for all the spacetime points, becoming a possible "universal constant" in Fantasia. The use of a global reference could be interpreted, in relativity terms, as a "view from infinity" of the information of correlation. From the most basic information of correlation among spacetime points, additional emerging features could arise, when considering more complex codes of aggregation and correlation among entities.

Within a cycle of $2 T$, we could consider as "current memories" all the information representing the potential non-local correlations in space, from which we could construct all the present space. We could call "Absolute Time" (or Tk for short) an ideal quantum buffer that stores the current potential as memories of correlation (after k-1 cycles) and from which, at each $2 k T$ space emerges. The adjective "Absolute" should not be intended in Newtonian terms but related to a preferred perspective and reference frame at infinity and to absolute references (as the "absolute zero" or the Planck units). The information of the past states in Tk could be related as well to a fundamental causal structure from which spacetime emerges, idea also elaborated in [13]. As we will see, both the causal structure considered in other models and Tk memory represent non-local hidden information evaluated through momenta in each elaboration cycle.

The Absolute Time memory, in its information processing evolution cycle, could identify a "global beat" that allows the coherent decoding of the spacetime fabric and, from a local perspective, the coherent decoding of the imaginary surroundings. It could be related to the concept of Quantum Potential (introduced in [14]) and could be seen as a delta of action at Planck scale that acts as a local reference for the Information Potential. 
In this context, we could consider the Planck constant $\hbar$ as the simplest absolute reference needed to properly define a relative confrontation in terms of unitary action. It is an absolute reference for a quantum of angular momentum, derived from the Planck units of mass, length and time, and could be interpreted as a "common beat", characteristic of the universe and consistent in every point in the emerging spacetime.

In a discrete evolution we could consider the concept of spatial distance within a given cycle deriving its physical meaning thanks to an absolute speed of light: we could define distance as "the imaginary time needed for light to cover that distance". If the speed of light were not an absolute reference for observers, there would be no coherent spatial distances and no perception of a coherent space. From the idea of an imaginary time, as the imaginary distance $i r$ between 2 points in empty space, we can construct an imaginary space considering, from any possible point as origin, the space described through ir and 2 degrees of freedom, in spherical coordinates. We could then consider the imaginary coherent time $i c t=i r=i n L=i L e^{\varphi}$ as the axis of a discrete spatial distance variable (with $c$ equal to the speed of light, $L$ the Planck length and being $i$ the imaginary unit). Note that in a flat space defined as "imaginary relativistic distances" through the imaginary coherent time, the conservation of causality accounts for coherence also in the "ticking" of the Absolute Time memory, adding the contribution (ict) $)^{2}$ to consider the causal information propagation in the imaginary space between 2 different points.

If there is entanglement, then "distance" should not only be considered through the propagation in a flat ict, but also through a logical connection in the Absolute Time Tk. It is as if spatial distance only had limited meaning giving the correlation in the memory axis of spacetime. Note that the non-local correlation between distant points is always related to a potential connected to past elaboration cycles (events) and cannot in any way allow faster than light communications. Actually, having required logical consistency on the information potential, causality violations are excluded a priori.

We could define the Coherent Now as the information of Tk emerging in ict in a full cycle. It could be interpreted as an absolute and coherent view from the current infinity on "what it was" (giving the cycles occurred) and a self-centered but impartial perspective on "what it could be" in ict (giving the freedom of choice within the current cycle and the past memories). What we call past in time is then, at least in Fantasia, the coherent connection of different events, occurred in previous cycles of Tk and already absolutely real in their consequences in the Absolute Time memory. What we call space seems then the connection of possible future coming information, developing along the imaginary time in a probabilistic way. The future, from a global and absolute perspective on the cycles of Tk, as already said, has not been written nor is real yet. Note that, from the local perspectives of observers in the universe, there is not a commonly perceived time flow speed (in Newtonian sense) nor an absolute spatial perception, as local measures of time and space distances are intertwined and shall be always considered relatively to the coherence with the speed of causality. Different observers may see events occurring in different order from their local and relative perspective, but events where already causally recorded in the Absolute Time at a specific cycle from the center of the event perspective and observers are just reading the causal memory from different angles.

The Coherent Now (pictured in Figure 1) could be interpreted as the thick now described in intuitionistic mathematic, as well as a philosophical "living present" we should all learn to appreciate and seize. It has a finite amount of information and comes as the $k^{\text {th }}$ cycle in the Absolute Time memory evolution since the birth of Fantasia.

At every cycle of Tk, Fantasia space emerges until the current possible infinity in ict (ict $\rightarrow \infty \mid \mathrm{k}$ intended as "just beyond the current radius @Tk" and not as a physical infinity). The Absolute Time memory, preserving the information of non-locality in space, allows the "unbroken wholeness of the entire universe" (Bohm et.al. in [15]). Note that evolution should not be reduced to simple deterministic automata rules. Memories influence the current state but does not define it completely. The current state depends as well on the still available degrees of freedom in the potential and on an evolving precision in intuitionist sense, preventing an absolute predictability of the future in each cycle. 
In the evolution of the spacetime information, as the current cycle passes, the information that collapsed becomes "absolutely real" in its consequences and what did not collapse keeps its potential. Eventually, as the first and ultimate "impartial observer" in Fantasia, the Absolute Time memory Tk records, elaborates, projects the information in ict and repeats its cycles through persistence (as a potential in a real memory) and transformation (in a probabilistic and emerging imaginary space).

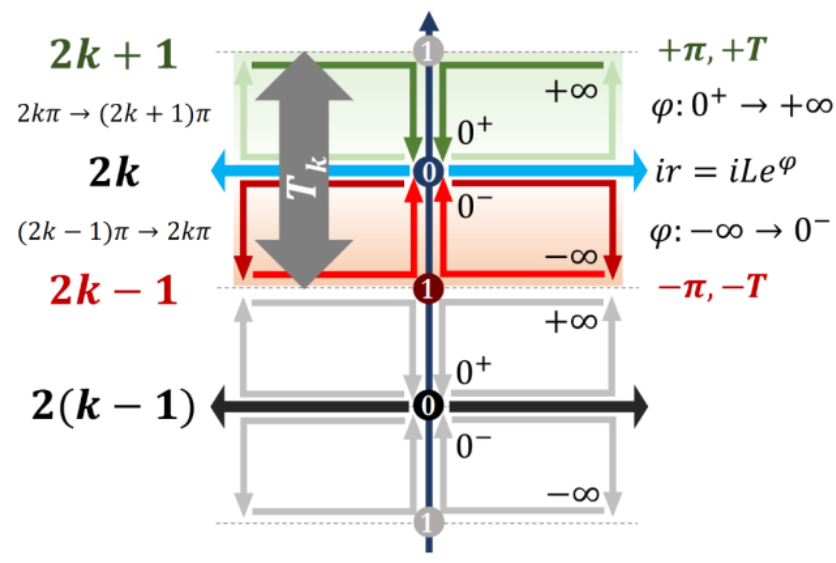

Figure 1. Schematic representation of the cyclic elaboration of the Absolute Time (Tk memory) at cycle (k-1) and (k). The imaginary space (noted as $i r$ ) emerges every $2 k T$ from the information of non-local correlations (kept as a potential in Tk). Events occurred up to (k-1) are considered as irreversible fixed points from which the current potential could evolve in the thick present at Tk.

\section{Memory-loops and log-perspectives}

To allow a consistent evolution and an emerging causality, information in the Coherent Now shall not be, as required in the hypotheses, under-determined (undecidable) or overdetermined (contradictory). Logical consistency is considered assured in Tk memory thanks to the combination of a forward and a backward-evolving wave function, existing as potential in the two hemicycles of the Coherent Now and related to the phase $\varphi$ developing in $(-\infty \mid \mathrm{k} \rightarrow 0-)$ and $(+\infty \mid \mathrm{k} \rightarrow 0+)$ respectively. In this context, the information in Tk that allows the emergence of a relative and causal spacetime fabric could be described through the concept of "memory-loops", intended as a potential related to closed time-like curves (CTC) within the thick present. Actually, loops are the most basic circuits for memory allocation in Information Science. In this context, CTC could be seen as connecting different points in the emerging space assuring logical consistency of the correlated information with a consistency-check forward and backward in ict through Tk within the Coherent Now. From this perspective, entanglement is equivalent to a CTC in the Coherent Now, described as a memory-loop of information potential stored in Tk. As illustrated in Fig.2, the memory-loops cross perpendicularly the spatial axis of the imaginary distances (as space and time orthogonality).

If we focus on space considering the points at $( \pm \infty \mid \mathrm{k})$ in $i c t$ as equivalent to a far distant origin in Tk of the information emerging as space, then ict could be pictured as on the boundary of Tk, also in line with an holographic description. In the context of a thick present, the perspective from both $-T$ and $+T$ could result in a dual-Anti-de-Sitter space, allowing the emergence of a flat and stable axis of imaginary space. The Absolute Time could be intended as a memory that elaborates the information of spatial correlation emerging in ict as in a neural network (idea also investigated in [16] and [17]). In this sense, Tk processing can be interpreted as well as a compression algorithm, able to account for all the complexities in the ict states, but further elaboration is needed.

From an info-compression perspective, a wavelet analysis (log-scale decomposition) could exploit the spatial scale invariances and fractal self-similarities of natural phenomena that usually follow a lognormal distribution (as highlighted by Kolmogorov in [18] and others in [19]), allowing the most efficient representation of the information in the imaginary axis of distance. 
In a parallel with ICT and traffic patterns, packets arrivals in telecommunication networks follow a lognormal distribution as well, due to a multiplicative nature of the variables involved (as discussed in [20]). The logarithm is also the function defining the information content and having a log-perspective seems more efficient for natural information elaboration. The Absolute Time is then intended, in the synthesis of the spatial information, as having a log-perspective on the correlation in ict (thanks to a wavelet analysis derived as an integral over the inverse of the ict distance), with shorter and local distances closer to the boundary and ict, while longer ones accounted deeper in the bulk memory of Tk.

Beside scale invariance, also shift invariance would be a desirable feature of an optimal compression algorithm of the spacetime information potential. The Dual-Tree Complex Wavelet Transform (DTCWT, described in [21]) could exploit both scale and phase shift invariance in the analysis and synthesis of the information emerging in ict from the potential in Tk. We could consider a DTCWT with an ideal filter bank transfer function $1 / t$ (acting as the real memory filter of Tk at different scales of ict) and the corresponding Hilbert transform pair $\delta$ (scanning the phase variations in the ortogonal imaginary space dimension) as a possible "compression algorithm" for the ict info in Tk.

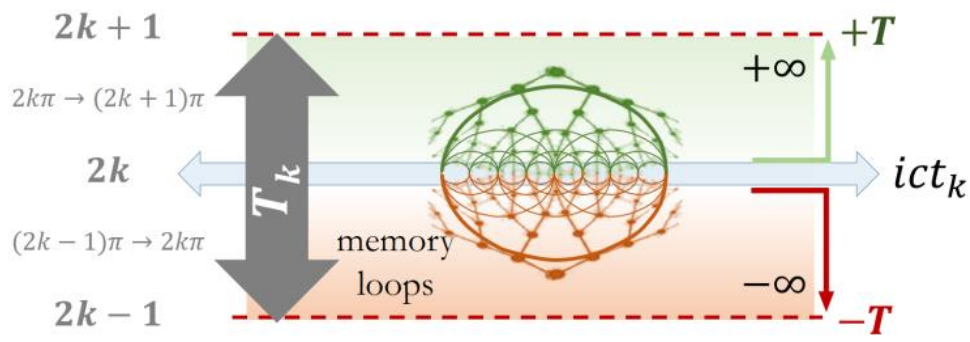

Figure 2. Coherent Now with ict imaginary spatial axis and Tk memory potential. The dual AdS description (from $-T$ to $+T$ ) results in a flat and stable emerging axis of imaginary space. Different scales of CTC (memory-loops) are represented, highlighting the log-perspective of the Absolute Time on the hidden information of non-local correlations in the imaginary axis of space.

\section{Synthesis of the model}

We started drafting a toy model of the universe named Fantasia and elaborated on how to define, bound and minimize the information needed to describe it. We have considered multiple perspectives on time, core element of any QG theory, and deduced the idea of a "thick present" as the only real information needed, considering time flow and space as both emergent from a more fundamental memory.

Summarizing, the main assumptions considered so far are:

- A simplified model of the universe (such as Fantasia) could be described through the analysis of the information correlation, given as finite, discrete, relative to global scales of reference and logically consistent

- Time, as an extended axis, is considered emergent. There is only the current instant, a fundamental "thick present" with all its associated potential of correlation, stored in a memory called Tk or "Absolute Time". The passage of time occurs following discrete elaboration cycles of the Tk memory. Each cycle is of duration 2T

- Space distance is considered as imaginary (ict axis) and emerges from the hidden information of correlation among distant imaginary points, stored and elaborated in Tk in each Coherent Now from $(2 \mathrm{k}-1) \mathrm{T}$ to $(2 \mathrm{k}+1) \mathrm{T}$

- The information of non-local correlations persists along the cycles of evolution as memory loops, which assure the logical consistency of the information. They represent a potential expressed in closed time-like curves (CTC) developing in ict @Tk

- The information of correlation could be summarized in Tk thanks to a log-perspective (that exploits the scale invariances and fractal self-similarities of natural phenomena) and a phase shift invariance in the imaginary axis of space ict, as in a DTCWT 


\section{Networks of momenta}

\section{Momenta and wave functions}

After having defined the engineering hypotheses and the high-level design of the considered model of the universe, it is worth focusing on elementary particles. We need to elaborate on how their information and evolution could be described in terms of the phase in Tk and ict, in both relativistic and quantum mechanical terms. The scope is to describe the Einstein 4-vector through the momenta in both Tk and ict and connect the wave function of an elementary particle to a vibrating mode in the Absolute Time.

In the current elaboration cycle, given a particle of relativistic mass $m$ that propagates in space with translational velocity $v_{s}$, consider the following equations:

$$
\beta=\frac{v_{S}}{c}=\sin \left(\tau_{s}\right) ; \quad \frac{1}{\gamma}=\sqrt{1-\beta^{2}}=\cos \left(\tau_{s}\right) ; \quad b=\beta \gamma=\tan \left(\tau_{s}\right)
$$

Consider then a momentum in time connected to the particle internal information. In a QM interpretation of the information potential, we could picture this momentum as a pulse connected to the often-called zitterbewegung (trembling motion) observed from the Centre of Mass (CoM) reference frame, different from $v_{s}$ that represents the translational velocity of the CoM in reference to an external frame of reference. Given the proposed variables and $E$ as the total energy, we can explicitly define the following momenta:

$$
\begin{aligned}
& p_{S}=\frac{E}{c} \sin \left(\tau_{s}\right)=\text { momentum in Space } \\
& p_{T}=\frac{E}{c} \cos \left(\tau_{s}\right)=\text { momentum in Time } \\
& E^{2}=\left(p_{T} c\right)^{2}+\left(p_{S} c\right)^{2}=\left(p_{S T}{ }^{*} c\right)\left(p_{S T} c\right)
\end{aligned}
$$

For a fermion of relativistic mass $m$ and translational velocity $v_{s}$, we can derive:

$$
\begin{gathered}
p_{T}=\frac{E}{c} \cos \left(\tau_{s}\right)=m c \cos \left(\tau_{s}\right)=m_{0} c \\
p_{S}=\frac{E}{c} \sin \left(\tau_{S}\right)=m \beta c=m v_{S}=m_{0} \gamma \beta c=m_{0} b c=b p_{T}=p_{T} \tan \left(\tau_{S}\right)
\end{gathered}
$$

For a photon, $\tau_{s} \rightarrow \pi / 2$ and, giving its frequency $f$, the considered momenta become:

$$
\begin{gathered}
p_{T}=\frac{E}{c} \cos \left(\tau_{s}\right)=\frac{\hbar f}{c} \cos \left(\tau_{s}\right)=\left(M \frac{f}{F}\right) c \cos \left(\tau_{s}\right) \rightarrow 0 \\
p_{S}=\frac{E}{c} \sin \left(\tau_{s}\right)=\frac{\hbar f}{c}=\left(M \frac{f}{F}\right) c \sin \left(\tau_{s}\right) \rightarrow\left(M \frac{f}{F}\right) c
\end{gathered}
$$

These momenta could be seen as driving, in each cycle, the evolution of a log-spiral associated to $e^{b \varphi}$ with $b=\tan \left(\tau_{s}\right)$. When $b=0\left(\tau_{s} \rightarrow 0\right.$ as for an ideal still fermion) the spiral becomes a circle (memory-loop), while it develops in a straight line when $b \rightarrow \infty\left(\tau_{s} \rightarrow \pi / 2\right)$ as for a propagating photon that has no rest mass and hence no static persisting information from the Absolute Time memory perspective. If we consider the momentum in space developing in a 3D imaginary Space defined by 3 orthogonal imaginary components, the momentum in time develops on the real axis, orthogonal to all 3 imaginary coordinates of space, as in Einstein 4-vector. In a simpler 1D-space we can see the momentum in space on the imaginary axis of space distance ict and the momentum in time on the orthogonal real axis, as a phase-info connected to the persisting information of the particle stored in the memory of Tk.

Beyond a relativistic description of the momenta, extending to $\mathrm{QM}$, consider the generic wave function (defined through amplitude and phase) describing the position of a massive particle. The amplitude $R$ is intended as the inverse of a length through the unit of measure $1 / L$, with $L$ equal to the Planck length.

$$
\psi_{E}=R e^{\frac{i S}{\hbar} ; R^{2}=\rho ;}
$$


We can define a quantum velocity $v_{q}$ derived from the Quantum Potential and connected to the internal degree of freedom within space and the spin of the particle (also proposed in [22] and [23]). We can consider $v_{q}$ as connected to the momentum in time defined before, seen as the particle pulse in time. For a fermion, given the relativistic description derived in Equation 5, we can consider this pulse equivalent to the speed of light in absolute terms (not intended as a massive particle travelling at the speed of light but more as "sampling frequency" of the spacetime information in Tk evolution).

$$
\left|v_{q}\right|=\hbar\left|\frac{\nabla R}{m_{0} R}\right|=c\left|\frac{M L}{m_{0}} \frac{\nabla R}{R}\right|=\mathrm{c}
$$

We can then define the momentum in time through the log-gradient of the amplitude of the wave function and the momentum in space as the gradient of the phase. The logarithmic gradient highlights the Absolute Time perspective on the information of spatial correlation persisting among the imaginary points of possible emergence in ict.

$$
\begin{gathered}
p_{T}=\frac{E}{c} \cos \left(\tau_{S}\right)=m_{o} c=M L c\left(-\frac{\nabla R}{R}\right)=-\hbar \nabla \ln (R)=p_{0}(-L \nabla \ln R) \\
p_{S}=\frac{E}{c} \sin \left(\tau_{S}\right)=m v_{S}=\nabla S
\end{gathered}
$$

Elaborating on the last equations, we can better express the relation between the particle's wave function and the momenta introduced.

$$
\begin{gathered}
\nabla \psi_{E}=\nabla e^{\left(\ln (R)+\frac{i S}{\hbar}\right)}=\left(\nabla\left(\ln (R)+i \frac{S}{\hbar}\right)\right) R e^{i \frac{S}{\hbar}}=-\frac{\left(p_{T}-i p_{S}\right)}{\hbar} \psi_{E} \\
\left(-p_{S T}{ }^{*}\right) \psi_{E}=\hbar \nabla \psi_{E} ; \quad p_{S T}{ }^{*}=-\hbar \nabla \ln \left(\psi_{E}\right)
\end{gathered}
$$

The spacetime momentum is connected to the Hamiltonian and the last equations could be related to the Schrödinger equation, where the gradient is intended over the emergence in the ict spatial dimension in a given elaboration cycle.

In the ideal absence of space momentum, a particle emerges at a distance $i r=i L e^{\varphi}$ from its CoM but most commonly at $i r_{c}$ (Compton radius, with a resulting equivalent wavelength $\lambda_{c}=2 \pi r_{c}$ ). From the relation of the Compton radius and the rest mass with the absolute scales of reference for length and mass, we could identify a characteristic dimensionless number or equivalent phase of the particle.

$$
\begin{gathered}
\frac{\lambda_{c}}{2 \pi L}=\frac{r_{c}}{L}=\frac{M}{m_{0}}=n_{c}=e^{\varphi_{c}} \\
-L \nabla \ln (R)=L \frac{p_{T}}{\hbar}=\frac{p_{T}}{p_{0}}=\frac{m_{0}}{M}=\frac{L}{r_{c}}=\frac{1}{n_{c}}=e^{-\varphi_{c}}
\end{gathered}
$$

In each cycle, the information of phase in $T k$ is related to the degree of freedom of the particle in its imaginary emergence in space (distance from the CoM), with associated potential cost and consequent apparent trembling and uncertainty. From a perspective centered on the CoM, we can describe the amplitude of the wave function as a Boltzmann distribution, connected to the equilibrium between the particle momentum in Time and the global pulse of the Absolute Time as well as to an emergence with maximum entropy.

$$
R=\frac{1}{r_{c}} e^{-\frac{r}{r_{c}}}=\left(\frac{p_{T}}{\hbar}\right) e^{-\left(\frac{p_{T}}{\hbar}\right) r}=\frac{1}{L} \frac{p_{T}}{p_{0}} e^{-\left(\frac{p_{T}}{p_{0}}\right) \frac{r}{L}}=\frac{1}{L}\left(\frac{1}{n_{c}} e^{-\frac{n}{n_{c}}}\right)=\frac{1}{L} e^{-\left(\frac{n}{n_{c}}+\varphi_{c}\right)}=\frac{R_{m}}{L}
$$

\section{The root of the matter}

To better picture how the momenta introduced relate with a description of a universe based on Tk and ict, we could describe an elementary particle as a local network of links, developing from the CoM and existing as a persisting and logically consistent information potential in the Absolute Time memory. We could consider the wave function as a packet of information representing these links between distant points from the particles' CoM. 
We could picture the particle emergence in the imaginary time as a trembling appearance in the probabilistic 3D Space, following these available links persisting in Tk. The correlation-links could be considered as bridges between the CoM and the imaginary points at distance $i r$ where the ideal point-like particle could emerge, extending $E R=E P R$ (Einstein - Rosen bridges $\leftrightarrow$ Einstein - Podolsky - Rosen "paradox" of entanglement) introduced in Holographic theories (as proposed in [24]) to the correlation among imaginary points in the emerging space of Fantasia at each cycle of Tk. Given that the correlation links persist in the memory of the Absolute Time as logically consistent memory-loops or $\mathrm{CTC}$, we can consider the following conceptual equation:

$$
E R=E P R=C T C @ T_{k}
$$

The energy-information parallel could be intended in the relation of a static mass to a gradient of correlation-info among imaginary points in space, persisting as a characteristic phase relative to the Absolute Time pulse.

The persisting information potential in Tk associated to the mass could be related to a local curvature in respect to a flat ict in empty space (when no additional non-local correlationlinks are present). The static-mass component of the stress tensor that curves the local geometry could be derived from a logarithmic gradient of the information potential in respect to the pulse of Tk. The correlation links are always well defined in the Coherent Now: even when no collapse occurred, the phase info is kept as absolute real potential, leading to a well-defined metric for gravity evaluation.

The wave function could be interpreted as the Root of Existence that connects mass, energy and information, as the root of a persisting information gradient. A point-particle manifests itself in the possible emergence at a point $i r$, but the particle is the ensemble of the correlation links, like a root in the memory of Tk that evolves as a packet of coherent information. The point-particle could be considered as just an event of collapse of the root potential in a localized past information at a given cycle of Tk. From a root in Tk, the event is a localized point ictelk where the potential in Tk manifested in ict and was recorded as a fixed point in the causal memory of spacetime (memory-knot).

Fantasia spacetime could be considered as emerging from a connected network and computational memory that elaborates and projects the full current space on a globally synched cycle. An elementary massive particle could be described as a network of hidden additional non-local correlations summarized in its Root of Existence. It could be seen as a local network in a greater Network, persisting in the memory of Tk as a smaller packet of coherently entangled imaginary distances. From Fantasia perspective, the ensemble of the correlation links related to the elementary particle identifies a volume of imaginary space on a common beat, like a local persisting tune. From an ICT perspective, the momentum in time could be interpreted as well as a self-decoding reference relative to the beat of the Absolute Time. In a wavelet decomposition of the potential in a local volume of space, it could be described as the local ground level of summarized information at the heart of the $\mathrm{CoM}$ and represents the coherent information common to all the imaginary points correlated in the same elementary particle, as well as the scale of the resonating wavelet. In this context, we could interpret the logarithmic progression of the masses of elementary particles as different scales of resonating wavelets and momenta each at proper depth the log-memory of Tk. The momentum in space could be described as additional information, which reduces the uncertainty in the direction of motion (expressed in the De-Broglie $\lambda$ ) and shapes the volume of possible emergence in the imaginary time.

Given the half spin of fermions, we can consider the evolution of massive elementary particles on a period of 2 cycles of Tk (equivalent to a $4 T$ or $4 \pi$ period). Fermions are then described by the information of the proper beat in the Absolute Time together with a memory of the information of self-orientation in ict in each cycle, intended as a quantum spin sign $s_{v}= \pm 1$ in the imaginary time. Entanglement among elementary particles in Fantasia relies then on the correlated information of opposite spin orientations, as a shift of half a period in the evolution of the memory bit $s_{v}$ to allow coherence of the global information 
potential (in line with the Pauli exclusion principle for particles entangled in the same atom, sharing the same CoM and on the same energy level).

We could consider antimatter as "opposite in the Absolute Time"-matter, which phases with inverted hemicycles along the elaboration in the Coherent Now. It could be interpreted as if the initial and final waves occurred along $\varphi$ developing in $(+\infty \mid \mathrm{k} \rightarrow 0+)$ and $(-\infty \mid \mathrm{k} \rightarrow 0-)$ respectively and the momentum in Time had consequently opposite sign in respect to matter. From this perspective, antimatter forward wave is developing in the Coherent Now in opposite direction in respect to the arrow of causality (as illustrated in Figure 4). We could metaphorically say that antimatter comes not from an underlying "Dirac see" but from the reference at $+T$ in respect to the emerging space in ict @2kT, as if dropping like a "Dirac rain" from the future and as if pulsing backwards in time in the Coherent Now (like a pendulum oscillating on an unstable equilibrium point). This potential exposure to greater instability could explain the asymmetry of antimatter abundance in respect to normal matter, in Fantasia as in our universe.

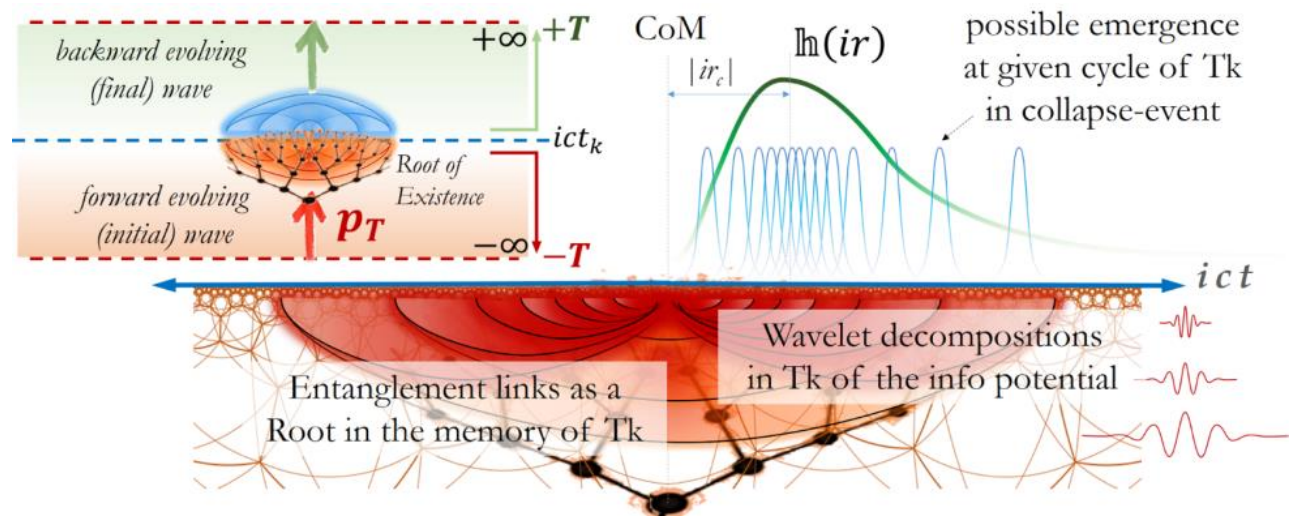

Figure 3. Schematic representation of an elementary particle as a network and root in Tk. In the top left corner, the full elaboration cycle is illustrated, highlighting the momentum in time and the memory-loops associated to the corresponding information in the initial and final wave functions. In the biggest picture, memory-loops (first hemicycle) are highlighted as a wavelet decomposition in the bulk of Tk. The possible emergence position in the ict spatial axis from the CoM is shown as light-blue pulses. The distribution of the possible points of emergence from the CoM (on the sphere of radius $i r$ ) is marked as $\mathbb{R}(i r)=4 \pi|i r R|^{2}$ and it is drawn in green.
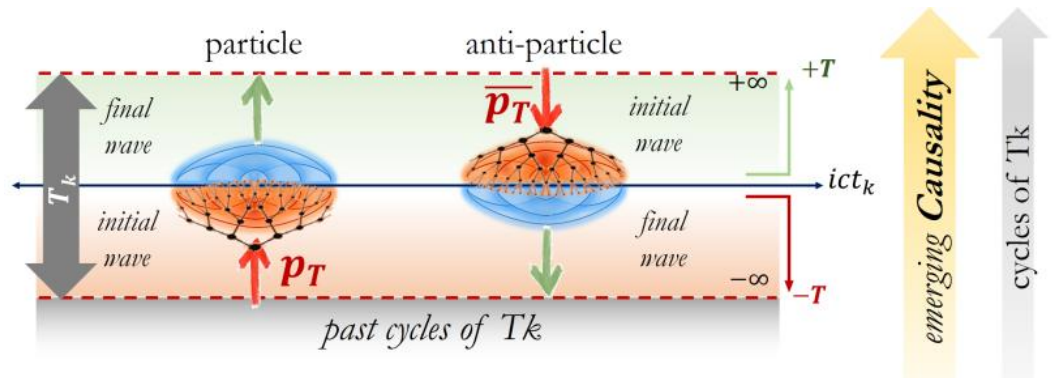

Figure 4. Schematic representation of an elementary particle and its corresponding anti-particle with inverted momentum in time. The wave function evolves on opposite hemicycles in the Coherent Now, as if propagating "backwards in time"

In this context, the force of gravity emerges from a real memory of persisting information related to the local additional correlation links. It is the information persisting as a potential in a fundamental memory that eventually becomes ontological. Given that the information in Fantasia is logically consistent, we could consider the information of gravity as coherent and causal on the evolution of Tk. Considering the ontological nature of the memory, gravity could be interpreted in this context as the "proof of existence" in the previous cycle of a static mass as a persisting information in spacetime.

As the coherence towards the Absolute Time reference is always perceived in an absolute sense, gravity is always attractive, independently from the sign of the momentum in time. 
The gradient persisting in the Absolute Time memory could be interpreted as a slope towards regions showing a "slower beat", proof of additional information in an absolute sense. Mathematically, the deviation from a flat spacetime due to a mass at distance ir could be derived thanks to a confrontation of perspectives between absolute and imaginary distances, identifying the "characteristic beat" at the local imaginary space point, as better illustrated in the dedicated Annex on Geometry.

\section{Few takeaways}

- In the proposed model, the Hamiltonian and the Schrödinger equation could be expressed in terms of a particle's momenta in the current cycle of elaboration. We could consider a momentum in time (associated to the persisting information in the memory of the Absolute Time) and an imaginary momentum in space (derived from the relativistic mass and the translational velocity in the imaginary time).

- The momentum in time depends on the static mass and it is derived from a logarithmic gradient of the amplitude of the wave function. It determines the emergence in space of the particle as a point at distance $i r$ from its CoM perspective (as a proper tempo in the emergence in $i c t$ ) and represents the scale of the resonating wavelet in the memory of the Absolute Time

- The wave function is related to the potential in the correlation links from the CoM to the point at ir where the particle could emerge, extending the holographic interpretation to $E R=E P R=C T C @ T k$. An elementary particle is the network of imaginary points and related correlation links represented by the wave function, real Root of Existence and vibration mode at given depth in Tk. In this context, the point particle is considered as an event of collapse in a given cycle at a specific imaginary point in ict, recorded as a fixed causal point (memory-knot) in the evolution

- Anti-matter could be interpreted as having a momentum in time with opposite sign and consequent opposite phasing in the hemicycles of the Coherent Now

- Gravity represents the memory of a local persisting gradient of correlation information in the cycles of Tk (due to a mass vibrating with proper scale on the beat of the Absolute Time). From an information perspective, it is the "proof of existence" of the rest mass in the previous cycle of Tk as a persisting information

\section{Conjectures on a physical implementation}

\section{Parallels on maximum entropy}

We have described the amplitude of the wave function of an elementary particle through a Boltzmann distribution, which maximizes entropy in the emergence along the imaginary time. The information of emergence from the CoM has been interpreted as the result of an equilibrium of the particle's momentum in time over the Absolute Time beat. In the proposed model, the momentum in time represents an optimal scalar compression of the persisting information of the static mass, defined through a log-perspective on the amplitude of the information, as a root and vibration mode in Tk.

In the limit of the mass equal to the Planck mass, the root of an ideal elementary quantum of information simplifies. It could be as if we were exploiting the maximum compression of information in a given quanta of spacetime, and consequently maximizing entropy (Shannon). Simplifying from Equation 15, we could derive:

$$
R_{1}\left(m_{0} \rightarrow M ; r_{c} \rightarrow L\right)=\frac{R_{M}}{L}=\frac{1}{L}\left(\frac{1}{n_{c}} e^{-\frac{n}{n_{c}}}\right)=\frac{e^{-n}}{L} \leftrightarrow n_{c}=1
$$

On a totally different scale, Black Holes $(\mathrm{BH})$ are as well entities showing maximum entropy, given the mass and the emerging surface at the horizon. Given $r_{s}$ as the Schwarzschild radius and $m_{b h}$ as the $\mathrm{BH}$ mass, we can consider:

$$
r_{S}=m_{b h} \frac{2 G}{c^{2}}=2 \frac{m_{b h}}{M} L=2 n_{b h} L
$$


As Black Holes maximally compress spatial information, we could consider a $\mathrm{BH}$ as an ensemble of $n_{b h}$ entangled quanta of maximum energy of $1 M$. The resulting wave function that maximizes entropy is derived combining orthogonally the elementary function of each quanta in a single Root and radius, with cumulate phase information and equivalent available memory (along the radius of the BH), as illustrated in Figure 5.

$$
R_{b h}=R\left(m_{b h}=n_{b h} M\right) \propto \prod_{x=1}^{n_{B H}} R_{M}=e^{-n_{b h} n} ; \quad \frac{m_{b h}}{M}=n_{b h}=\left|\nabla \ln \left(R_{b h}\right)\right|
$$

Both black holes and elementary particles seem following similar behaviors of maximum entropy and maximum compressed information. Elementary particles maximize the entropy in the spatial emergence, while maximum compression of information is obtained in the Absolute Time memory of Fantasia. Black holes maximally compress information in space and shows maximum entropy on the horizon. The parallel on similar behavior could offer insights on a possible connection between the 2 phenomena, as further elaborated in the following chapter.

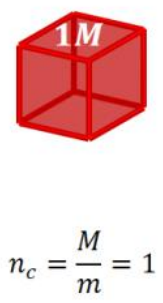

(a)

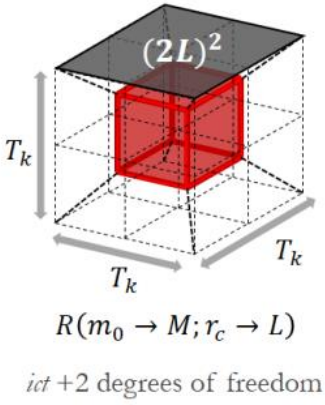

(b)

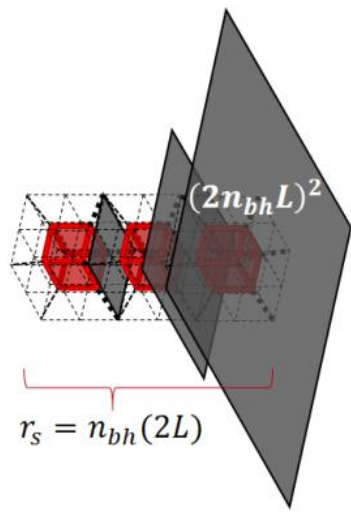

(c)

Figure 5. Schematic representation of an elementary quantum of mass (a) and its corresponding possible degrees of freedom as a single qubit memory (b). The entanglement of several elementary quanta in a single root is illustrated in (c), highlighting the resulting cumulative $\mathrm{BH}$ radius and the corresponding surface at the horizon.

\section{The Black Hole perspective}

We could picture a $\mathrm{BH}$ as the result of entangled quanta of maximally compressed information behaving as a coherent and independent entity. It appears indecipherable from the surrounding universe and only seems to show global or summarized properties. From the outside, we see a black body showing maximum curvature in ict, as a gradient tending to a delta and equivalent to a "step down" in ict that even light cannot overcome in the opposite direction. On the horizon, the spatial dimension (imaginary time) could be seen as "sinking" vertical in the Absolute Time. In the fall into a BH, absorbed particles are hence decomposed, filling elementary bits of maximum compressed information.

From a telecommunication perspective, the surface of the $\mathrm{BH}$ could be interpreted as a patch of spacetime shifted to $-T$ due the delta discontinuity in ict. In terms of information decoding, the instant shift of $1 T$ is equivalent to seeing the $\mathrm{BH}$ spacetime inner patch as in a decoder eye-diagram on the wrong sync (and so as incoherent and indecipherable).

Following the parallels on entropy, we could relate the information in a $\mathrm{BH}$ to a hypothetical inner universe (such as Fantasia) emerging beyond its singularity.

The radius could be mapped to the Absolute Time memory and the compact information at the horizon could be considered as equivalent to a summarized view from infinity of the inner imaginary time ictin emerging at the singularity, as illustrated in Figure 6 and 7.

The collapse to a $\mathrm{BH}$ could be equivalent to an inflation era from the ictin perspective: where the outside spacetime information in ict collapses (in an entangled line of quanta as a radial coordinate of the $\mathrm{BH}$ and root) the internal imaginary time ictin exponentially expands. 
In the proposed model, before the "bang", the birth of Fantasia could metaphorically be interpreted as the firsTk1ss of quanta full of possible persisting memories that entangle as a single root on a common beat. From the firsTk1ss, as the first thick tick $T_{1}$ and seed of a new real memory and Absolute Time, a network of information could evolve coherently as a new patch of spacetime and independent child-universe.

In the BH inner universe, elementary massive particles could be mapped to specific vibration modes with given $1 / n_{c}$ in the radius of the $\mathrm{BH}$ : each quanta would resonate on its proper wavelet scale, becoming equivalent to the quantum field of the corresponding particle, superposed with different amplitude of probability on different phases along the ictin space (as illustrated in Figure 8, 9 and 10). Considering the widest and deepest possible wavelet in the given radius of the $\mathrm{BH}$, we could as well give a physical interpretation to the concept of "minimum mass" in a universe and the idea of a quantum of gravitational information.

Natural units of the inner universe could be defined after the collapse event, depending on the number of quanta entangled in the root, in a gravitational lensing towards smaller scales, keeping the elaboration cycle constant in all universes.

$$
\begin{gathered}
T_{\text {in }}=T_{\text {out }}=T=\frac{1}{F} ; \quad L_{\text {in }}=\frac{L_{\text {out }}}{n_{\text {bh }}} ; \quad M_{\text {in }}=M_{\text {out }} n_{\text {bh }} ; \\
c_{\text {in }}=\frac{c_{\text {out }}}{n_{\text {bh }}} ; \quad \hbar_{\text {in }}=\frac{\hbar_{\text {out }}}{n_{\text {bh }}} ; \quad G_{\text {in }}=\frac{G_{\text {out }}}{n_{\text {bh }}{ }^{4}} ;
\end{gathered}
$$

The genesis, from BH collapses, of inner imaginary universes with proper memory and characteristics (in terms of natural constants and particles' mass in the inner universe) could be related to a possible cosmological natural selection, in a fractal implementation of the offspring as maximum compressed and hidden information within the memory of the parent universe.

In this context, elementary bosons could be related to the information needed to allow synched interactions in a connected network: virtual messages able to give a dynamical tense to static masses' possible events or actions in respect to the Coherent Now (like verbs in a sentence). Gravity could be intended as a message communicating the proof of existence already introduced, like an "I was" propagating on the horizon with long range and interconnecting in the shortest ranges the spacetime fabric at infinity; gluons could be related to a "I $\mathrm{am}^{\prime}$ " message, indicating orientation of quarks and hadrons for a coherent interconnection on the same root at infinity, and propagate in the bulk on short distances only; weak bosons could be picture as a "I'm going to be" message, carrying in Tk the massenergy for the communicated transformation among elementary particles; photons could be interpreted as the "I' $m$ being", connected to a message expressing the charge, as a dynamical transformation in the ict axis on the boundary at the singularity (an so propagating on long distances); finally the Higgs field could be related to an information of static, like a "I have" expressing the interaction of mass in the memory as a local selfanchor and consequent inertia.

The parallel between bosons and verbs giving a dynamical tense within the Coherent Now could be interpreted as a metaphor as well as a possible physical interpretation of forces as messages for the communication of events and actions in an interconnected universe. Different messages propagate at different depth in the Absolute Time and have consequently different ranges, as further illustrated in Figure 10.

Considering the evolution of a universe, the growth of the $\mathrm{BH}$ mass could be interpreted, from the ictin perspective, as an accelerated expansion, drifting apart regions of the inner imaginary space faster than the coherent given time. The growth seems logical to occur on the largest scales of the inner universe, adding new possible wider vibration modes in the memory. In terms of conservation laws, from the perspective of the universe hosting the $\mathrm{BH}$, energy seems conserved (following mass-energy-info equivalence in the Absolute Time) but local phases in space (as local distances among and within particles in the imaginary space ict@Tk) may be lost in the maximum compression of information. 
This conjecture could offer as well possible insights on the experimental discrepancies of the Hubble parameter, seen in the measurements via supernovae or through the Planck satellite (reported in [25]), as the 2 methods analyze data from different periods in the evolution of the universe and the mass absorption rate could have been different.

Further details on the possible interpretation of the external mass falling into the parent $\mathrm{BH}$ as the cause of Dark Energy variations in the inner universe, as well as an analysis of the dynamics of space expansion in the model are collected in the dedicated Annex.

The following figures schematically illustrate the concepts proposed, identifying the main elements of the model from both the outer $\mathrm{BH}$ and the inner-universe perspectives.

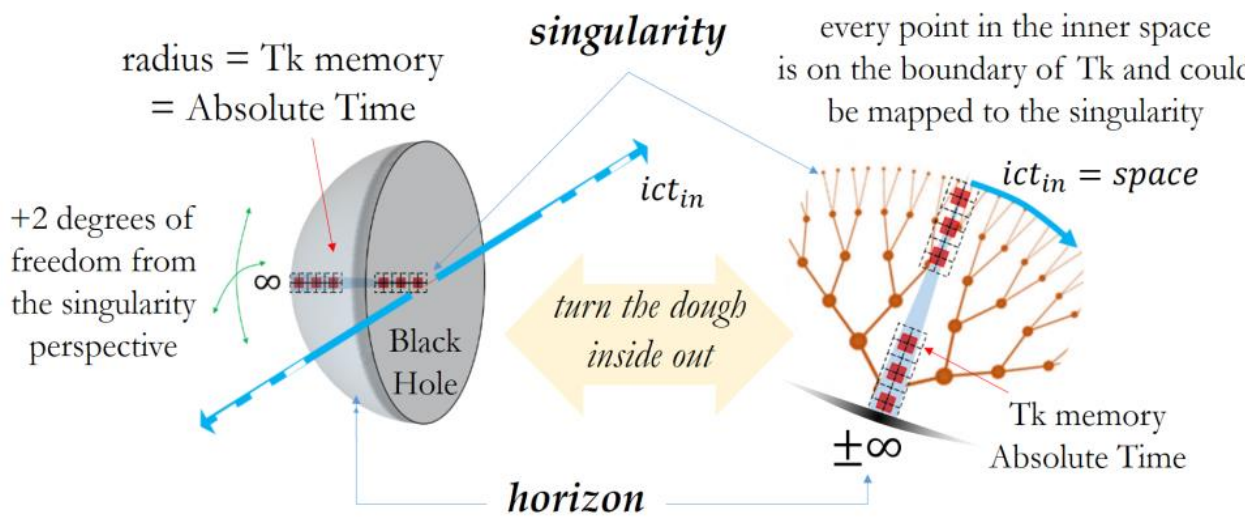

Figure 6. Schematic representation of the modelled universe as a $\mathrm{BH}$. The radius represents the Absolute Time memory of the inner universe imaginary time (emerging beyond the singularity as an imaginary distance $i c t_{i n}$ ). Both the $\mathrm{BH}$ radius and the inner $i c t_{i n}$ have 2 additional degrees of freedom, leading to a 3D imaginary space and corresponding memory. The horizon represents the far point at infinity on the given direction, while the singularity any possible point in the shift invariant ictin

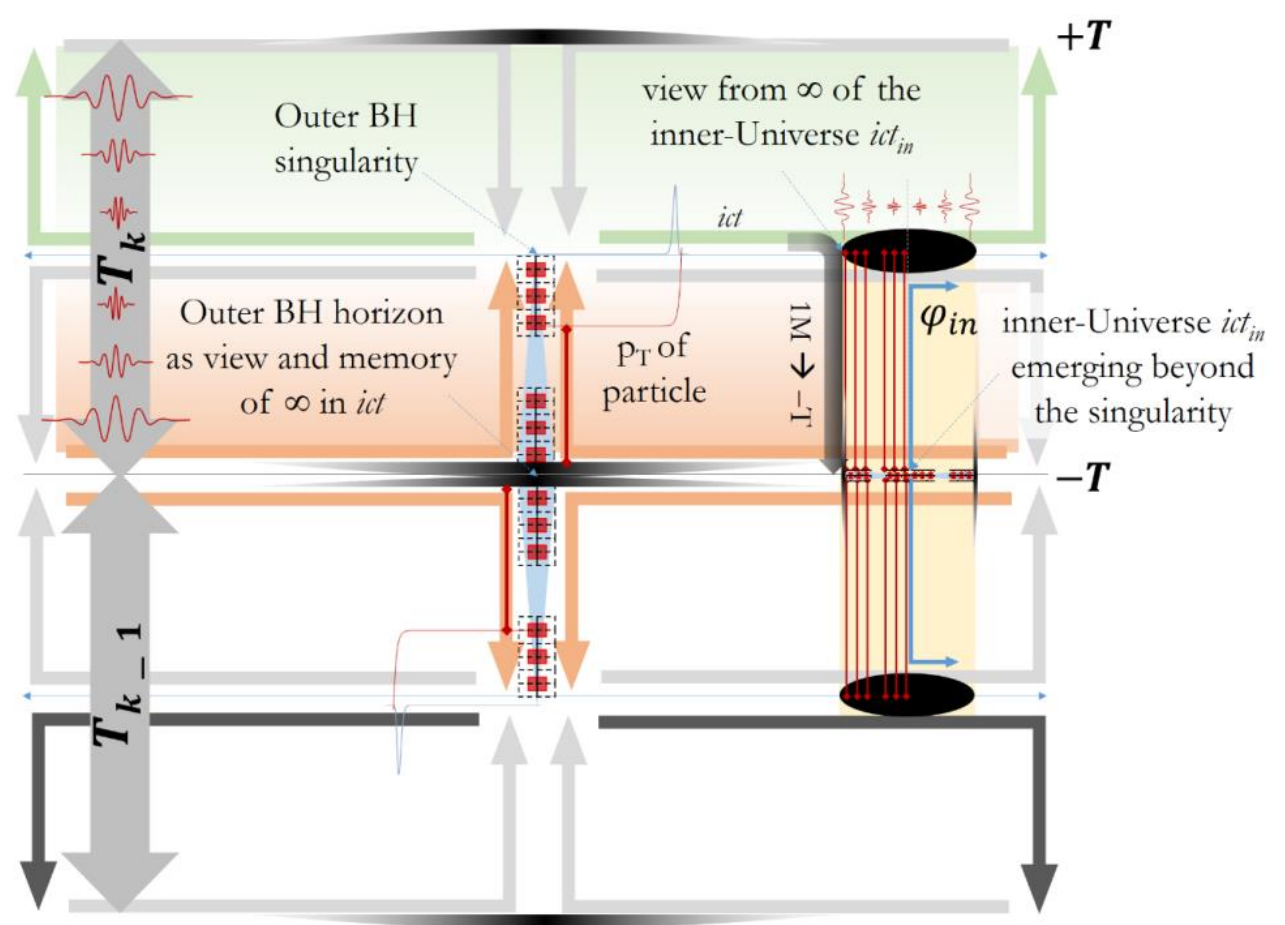

Figure 7. Schematic representation of a inner-universe and the corresponding parent $\mathrm{BH}$ as described from the perspective of the universe in which the $\mathrm{BH}$ is formed. The imaginary space dimension in the outer universe sinks to $-T$, following the delta discontinuity on the horizon. 


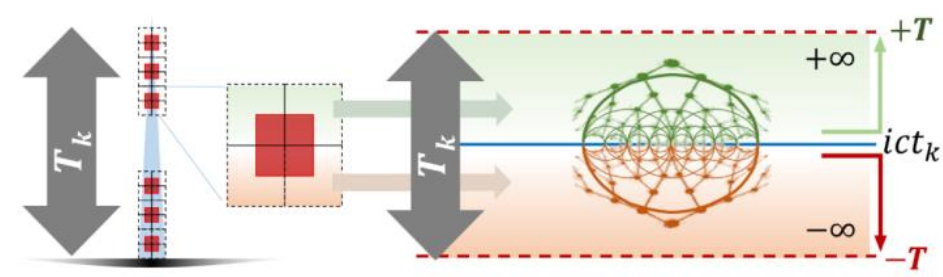

Figure 8. The $\mathrm{BH}$ radius is composed of ordered qubits, which represent the correlation between distant imaginary points in the emerging spacetime beyond the singularity. Persisting information of imaginary distance is described in each quantum with a perspective from $-T$ and $+T$. Resulting ictin comes from the superposition of the potential in each qubit, which could be described as a memory-loop or a CTC in the Coherent Now.

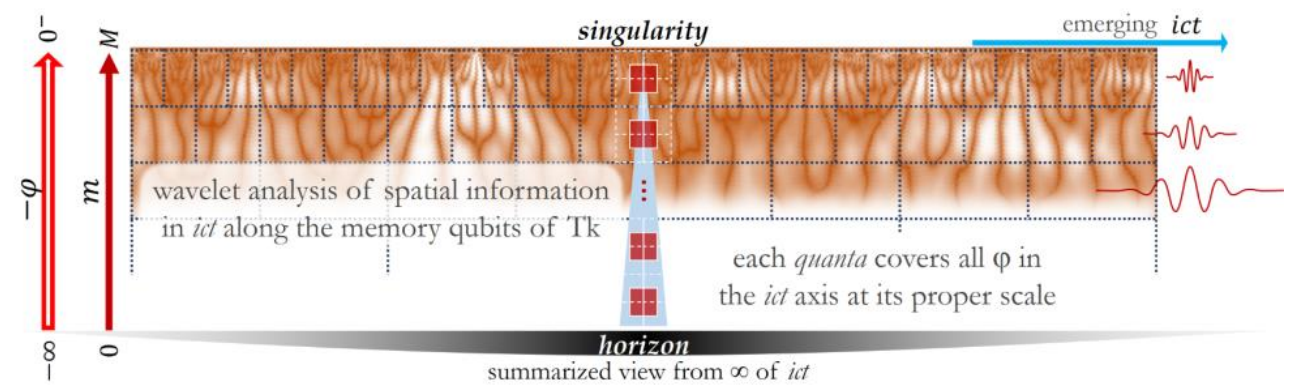

Figure 9. Each quantum in the $\mathrm{BH}$ radius vibrates at proper scale in the emerging space, in a gravitational lensing from the scales of the outer universe to the scales of the inner imaginary time. The correlation in ictin could be seen as decomposed thanks to a complex wavelet transform, which offer an efficient log-perspective as well as a shift invariance in the emerging imaginary space.

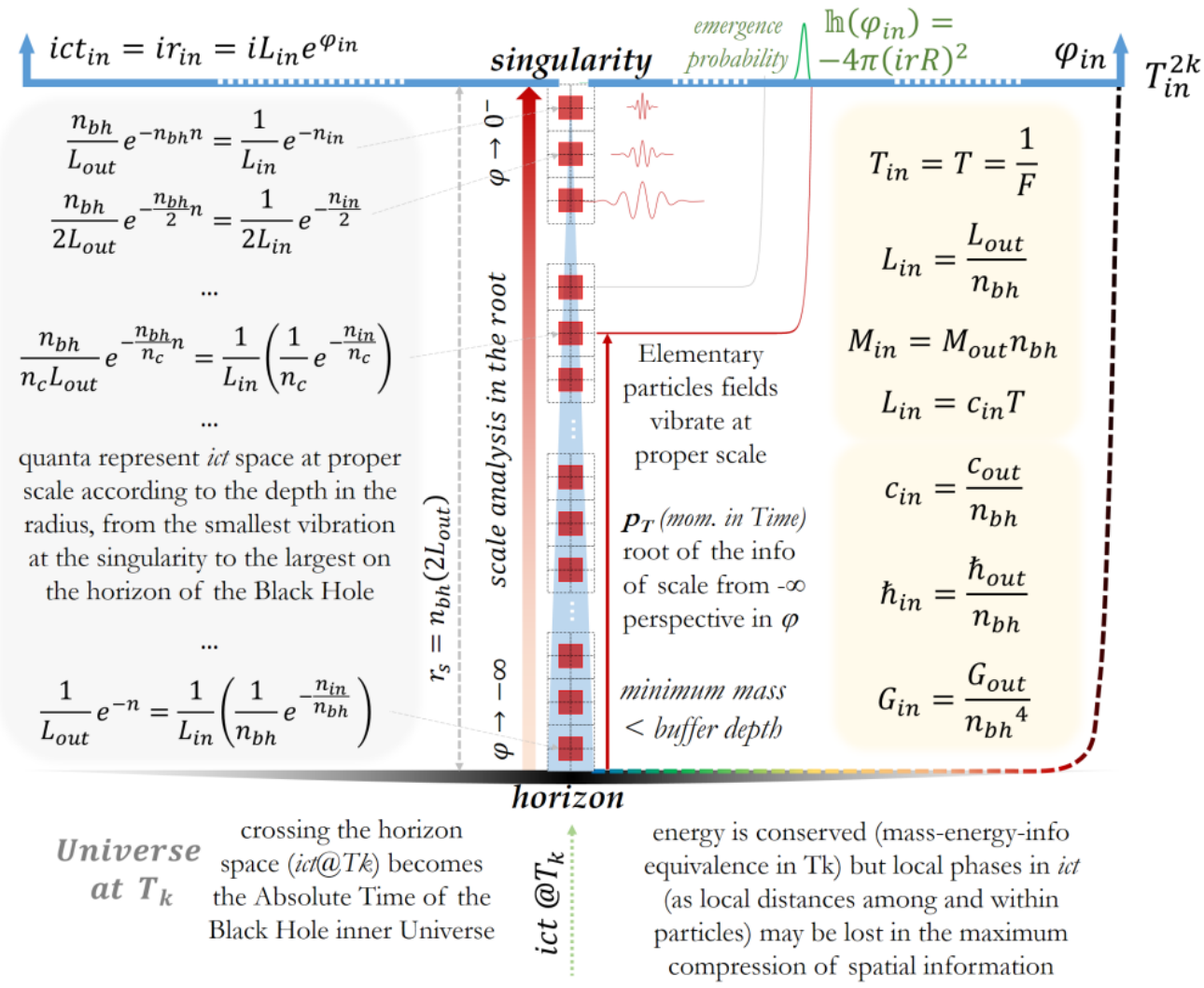

Figure 10. Representation of the vibration modes in the $\mathrm{BH}$ radius as elementary massive particles in the emerging imaginary universe. Natural units and universal constants of the inner universe depend on the radius of the parent $\mathrm{BH}$ at the collapse event and singularity formation (birth of the inner universe in the firs Tk1ss, as the first coherent vibration and $\mathrm{T}_{1}$ of its Absolute Time memory) 


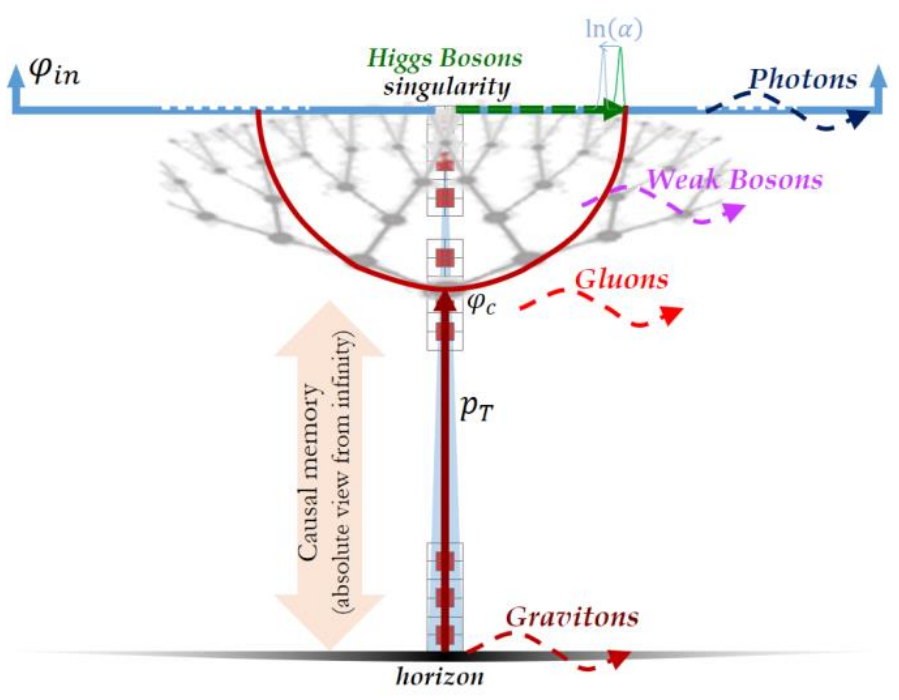

Figure 11. Representation of elementary forces as messages at different depth in the $\mathrm{BH}$ radius giving a dynamical tense (as verbs) to the evolution of matter particles. The Higgs Boson (I HAVE) is an information of static (as anchor of the root to the singularity) and consequent inertia in the imaginary time freedom of action; Photons ( $I^{\prime} m$ BEING) indicates occurring transformation (as local additional charge in each cycle) and propagates on the boundary at the singularity (decreasing as $1 / r^{2}$ ); Weak Bosons (I'M GOING TO BE) carry info of mass-energy of a coming transformation (as a cut or graft of branches), propagate in the bulk of Tk and hence act on short distances (on the same global origin at infinity); Gluons ( $A M$ ) represent information of orientation in respect to Tk, enabling the entanglement of quarks and hadrons on a single root, propagate in the bulk and act on short distances; Gravitons (IWAS) carry information of persisting existence, propagate on the boundary at the horizon (decreasing as $1 / r^{2}$ ) and along the $\mathrm{BH}$ radius

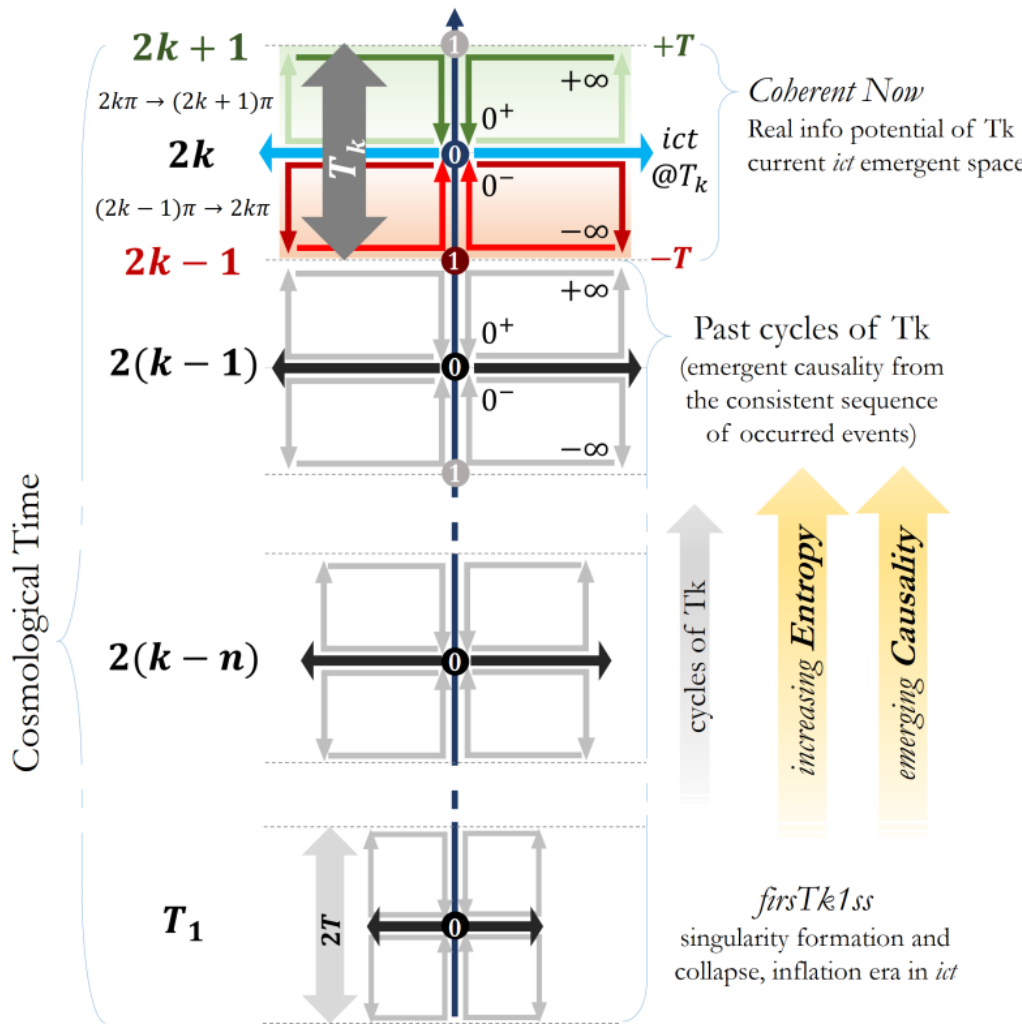

Figure 12. Different arrows of time as emerging in the model, from the birth of the new universe in the collapse of a BH (firsTk1ss) and the formation of its Absolute Time memory to the current instant (Coherent Now). Evolution proceeds in discrete evolution cycles of duration 2T. The total duration of all occurred cycles represents the inner universe cosmological time 


\section{Discussion}

\section{Synthesis of the proposal}

Starting from an Information and Communication Technologies perspective focused on implementability, we have proposed a model of the universe in which spacetime could be described through the analysis of correlated information. We have deduced the need for discrete and finite information, as any mathematical infinity would need an unrealistic infinite time and memory to be described. We have required logical consistency of the information potential (as the need for coherence among local perspectives and a global description of the information) and inferred the need for global scales of reference.

Following a Presentism approach and a discrete evolution cycle, we have considered a thick present as the only real information to be accounted, persisting in a fundamental causal memory called Absolute Time (Tk). In the proposed model of the universe, the information of hidden non-local correlations within the thick present persists as a potential in logically consistent CTC (memory-loops), summarized in the Absolute Time through a logarithmic perspective (able to exploit the fractal self-similarities of natural phenomena). The Absolute Time memory represents the real basis on which an imaginary coherent time ict may develop as a probabilistic spatial distance axis, still rich of degrees of freedom at proportional cost and undetermined future in each evolution cycle.

We have expressed the equivalence of mass and energy through the momenta along both Tk and ict and proposed the description of elementary particles as networks of bridges from the CoM reference frame, extending the holographic principle to ER=EPR=CTC@Tk. The rest mass has been connected to a momentum in Time (in Tk), as a persisting information of a proper tempo in the emergence in ict in respect to the reference beat of the Absolute Time. The momentum in Time could be intended as a "common beat" or a phase in Tk shared by the imaginary points belonging to the particle wave function and represents the persisting information of the particle along the evolution cycles as well as the scale of the resonating wavelet in the log-memory of the Absolute Time.

We have described the force of gravity as the result of a gradient in the information potential, manifesting as a gradient in time towards area showing a slower beat in respect to the Absolute Time reference. In this context, gravity has been proposed as a "proof of existence" in the fundamental causal memory introduced, which eventually becomes as well ontological.

Finally, inspired by a parallel on entropy and compression of spatial information, we have proposed a physical realization of the model as an independent patch of spacetime emerging beyond the singularity of a $\mathrm{BH}$, as its inner imaginary universe. We have proposed a possible mapping of the $\mathrm{BH}$ radius to the Absolute Time memory, deriving the global references of the inner universe (universal constants) in a lensing towards smaller scales, from the horizon to the singularity. Elementary quantum fields have been described as vibration modes at different depth in the $\mathrm{BH}$ radius, from the shortest wavelets closer to the singularity to the widest near the horizon.

The model of the universe presented would like to offer a possible perspective on spacetime that restores the value of time and memory in the physics' narrative, highlighting the importance of logical consistency in all theories based on information. Further elaborations are needed for an accurate description of entities' existence and evolution, as well as for an explicit reduction of the model to GR and QFT. Nevertheless, the framework identified offers a coherent description of a simplified universe and the derived insights could be useful steps towards a more fundamental theory of spacetime based on information.

\section{Open questions}

The proposed model offers a coherent description of a simple universe based on information, but still several aspects need to be further elaborated. Moreover, it is worth investigating possible connections between the framework introduced and the concepts of consciousness and evolution. 
To mention a few of the still open points, we could consider:

- explicit reduction of the model to GR and QFT

- early universe size and minimum memory for elementary particles existence

- particles' mass in relation to parent black hole radius

- description of dark matter

- fine structure constant in relation to the geometry of the parent black hole

- derivation of the "summary at infinity" at the parent black hole horizon

- quantum gravity phenomena at atomic and molecular scale

- experiments for particles' decay through induced decoherence of the root in Tk (towards a possible distributed quantum energy production)

- description of consciousness as a logical consistent access to a memory

- analysis of evolution as cycles of persistence and transformation of potential communication codes at different levels of abstraction

\section{Acknowledgements}

The author would like to thank Alexis Tsoukiàs (Research Director at the National Center for Scientific Research CNRS in France and beloved uncle) and Carlo Pellacani (Retired Professor of Physics and Cosmology at the University of Bologna) for the encouragements on the development of the proposal.

A huge thanks to the participants of the TM-2019 conference on QG, where the first ideas were presented $^{1}$, and in particular to Eliahu Cohen (Senior Lecturer at the Bar Ilan University) for the consultation on the time symmetric approaches to QM. The author is also very grateful to the QISS community ${ }^{2}$ for the fruitful discussions on a quantum description of our universe based on information, from both a physical and a philosophical perspective.

Finally, the greatest thanks to my family and friends for their support in this wild journey.

\section{References}

[1] Ä. Baumeler, "Causal Loops: Logically Consistent Correlations, Time Travel, and Computation," PhD Thesis, Università della Svizzera Italiana, 2017.

[2] N. Gisin, "Indeterminism in Physics, Classical Chaos and Bohmian Mechanics. Are Real Numbers Really Real?," Erkenn, 2019.

[3] V. Vedral and C. Marletto, "Gravitationally-induced entanglement between two massive particles," Phys. Rev. Lett., vol. 119, no. 24, 2017.

[4] C. Rovelli and M. Christodoulou, "On the possibility of experimental detection of the discreteness of time," Front.in Phys., vol. 8, 2018.

[5] N. Margolus, “Finite-State Classical Mechanics," RC, 2018.

[6] G. Jaroszkiewicz, Quantized Detector Networks: The Theory of Observation, Cambridge University Press, 2017.

\footnotetext{
1 TM2019, Turin - Italy. Visit indico.ict.inaf.it/event/751/ for further information

${ }^{2}$ Quantum Information Structure of Spacetime. Visit www.qiss.fr for further information
} 
[7] T. C. N. Boekholt, S. F. Portegies Zwart and M. Valtonen, "Gargantuan chaotic gravitational three-body systems and their irreversibility to the Planck length," Monthly Notices of the Royal Astronomical Society, vol. 493, no. 3, 2020.

[8] S. Watanabe, "Symmetry of physical laws. Part III. Prediction and retrodiction.," Reviews of Modern Physics, vol. 27, p. 179-186, 1955.

[9] E. Cohen, M. Cortes, A. C. Elitzur and L. Smolin, "Realism and causality I: Pilot wave and retrocausal models as possible facilitators," Phys. Rev. D, vol. 102, no. 12, 2020.

[10] E. Cohen, M. Cortes, A. C. Elitzur and L. Smolin, "Realism and causality II: Retrocausality in energetic causal sets," Phys. Rev. D, vol. 102, no. 12, 2020.

[11] L. J. Henderson, A. Belenchia, E. Castro-Ruiz, C. Budroni, M. Zych, C. Brukner and R. B. Mann, "Quantum Temporal Superposition: the case of QFT," Phys. Rev. Lett., vol. 125, 2020.

[12] N. Gisin, "Classical and intuitionistic mathematical languages shape our understanding of time in physics," Nature Physics, vol. 16, pp. 114-116, 2020.

[13] L. Smolin, “The dynamics of difference," Found Phys, vol. 48, 2018.

[14] D. Bohm, "A suggested Interpretation of the Quantum Theory in terms of "hidden" variables," Physical Review, vol. 85, no. 2, 1952.

[15] D. Bohm, B. Hiley and P. Kaloyerou, "An ontological basis for the Quantum Theory," Physics Reports, no. 6, pp. 321-375, 1987.

[16] P. Caputa and J. M. Magan, "Quantum Computation as Gravity," PHYSICAL REVIEW LETTERS, pp. 122, 231302, 2019.

[17] V. Vanchurin, "The world as a neural network," Entropy, vol. 22, no. 11, 2020.

[18] A. N. Kolmogorov, “On the logarithmic normal distribution law of particles with dimensions of fragmentation," Dok. Acad. Nauk SSSR, vol. 31, p. 99, 1941.

[19] E. Limpert, W. A. Stahel and M. Abbt, "Log-Normal distributions across the Sciences: Keys and Clues," BioScience, vol. 51, no. 5, pp. 341-352, 2001.

[20] I. Antoniou, V. V. Ivanov and P. Zrelov, "On the log-normal distribution of network traffic," Physica D, vol. 167, p. 72-85, 2002.

[21] I. Selesnick, R. Baraniuk and N. Kingsbury, "The Dual-Tree Complex Wavelet Transform," IEEE SIGNAL PROCESSING MAGAZINE, Vols. 1053-5888/05, 2005.

[22] S. Esposito, “On the role of Spin in Quantum Mechanics," Found Phys Lett, vol. 12, p. 165-177, 1999.

[23] G. Salesi, "Spin and Madelung fluid," Modern Physics Letters A, vol. 11, no. 22, 1996.

[24] J. Maldacena and L. Susskind, "Cool horizons for entangled black holes," Progress in Physics, 2013.

[25] K. C. Wong, "H0LiCOW XIII. A 2.4\% measurement of H0 from lensed quasars: 5.3 $\sigma$ tension between early and late-Universe probes," Monthly Notices of the Royal Astronomical Society, vol. 498, no. 1, 2020. 


\section{Annex on Geometry}

\section{Riemann and Apollonius}

The following Annex would like to offer a better understanding of the variables involved and their relationship with the gravitational information. In the model of the universe proposed, we have introduced a description of spacetime based on the information of correlation among distant points, stored in a computational memory from which space emerges as imaginary, along the axis ict, in each elaboration cycle.

The persisting information is stored as a momentum in Time, derived through confrontation with absolute scales of reference and related to an equivalent proper beat (as a resonating scale in ict). This momentum relates the static mass of the particle to the probability of emergence in a given point in space in ict (described in the wave function) and could be seen as orthogonal to the imaginary ict spatial axis, in a resulting complex spacetime plane. The use of absolute references has been related to the need of relativity with the perspective at infinity on the non-local and hidden information of correlation. This is equivalent to the requirement of a global logical consistency in the information stored in the Absolute Time memory.

Considering ict as the imaginary spatial axis of a spacetime plane (with the relativistic time on the real orthogonal axis), we can picture a synthesis of the emerging information in the circle of unitary radius centered in the origin, while the emerging universe becomes mapped to the outer plane, including infinity. To offer a geometrical description of the complex plane considered, we can refer to the Riemann sphere, a model to extend the complex plane that includes the point at infinity. Through the Riemann sphere it is easier to picture the relationship between a perspective at infinity and a perspective centered on the self $(\mathrm{CoS})$ of the same information, identifying the point $P$ and $P^{\prime}$ and their corresponding projections $A$ and $A^{\prime}$, as illustrated in Figure 13.

The geometry introduced in the Apollonius circle (illustrated in Figure 14) could help identifying the points $A$ and $A^{\prime}$ as the stereographic projection of $P$ to the imaginary axis ict. In both Riemann projection and Apollonius representation of the proposed model, the point $O$ represents the horizon of the $\mathrm{BH}$, far origin at infinity of the imaginary space, while the point $I$ represents the singularity from which the imaginary space develops. The segment $\mathrm{OI}$ (radius of the sphere) is related to the Absolute Time memory and the $\mathrm{BH}$ radius. From a perspective at infinity, OI maps the information as an inverted mirror of the emerging space from $I$ to infinity.

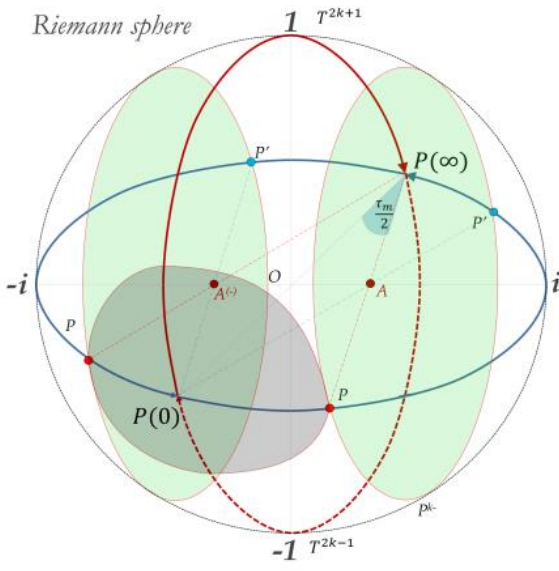

(a)

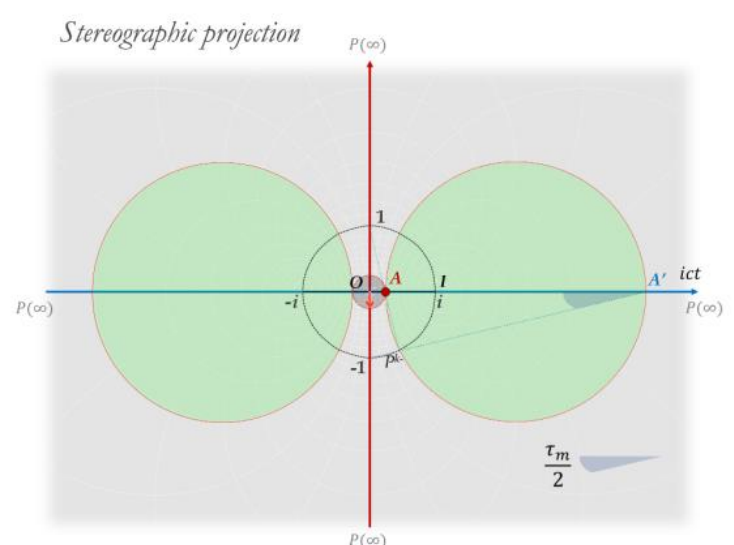

(b)

Figure 13. The Riemann sphere as a representation of the complex plane including the point at infinity is showed in (a) and its stereographic projection from the point $P(0)$, as Center of the Self $(\mathrm{CoS})$, on the complex plane is showed in (b). From $P(\infty)$ on the sphere, a point $P$ is projected to the point $A$ in the space identified by the segment $O I$, while it is mapped to $A^{\prime}$ on the space from $I$ to infinity when projected from the $\operatorname{CoS}$ at $P(0)$. 


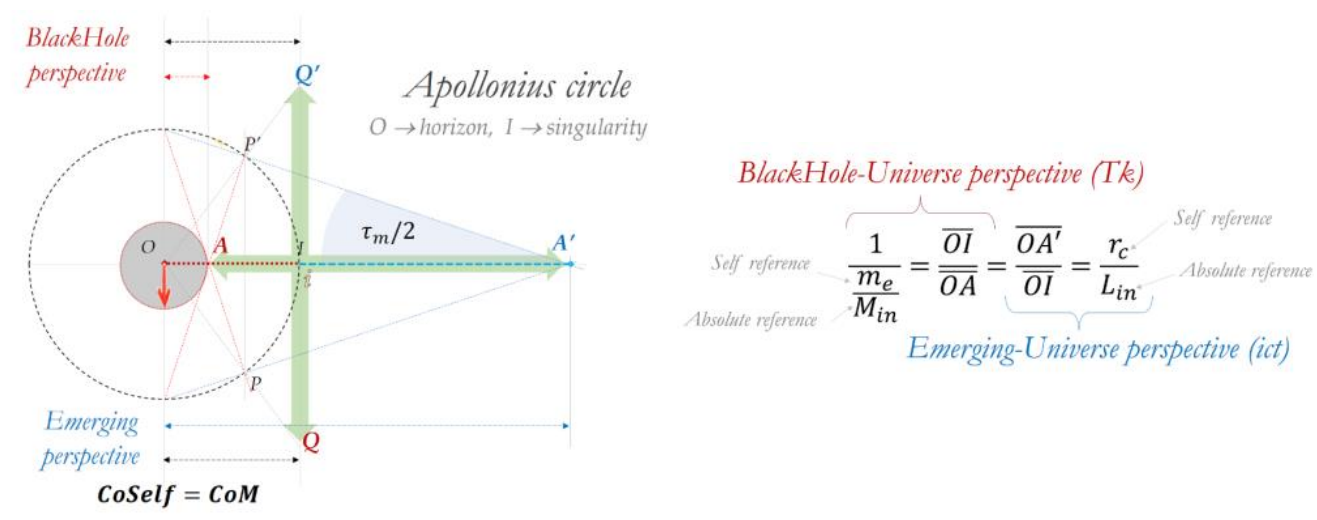

Figure 14. The geometric relations between the distance of the projections $A$ and $A^{\prime}$ from the point $\mathrm{O}$ at the origin (horizon of the parent $\mathrm{BH}$ ) is described through the Apollonius circle, highlighting the perspective on information in Tk (BH-universe) and ict (imaginary emerging-universe inside the $\mathrm{BH}$ beyond the singularity at $I$ ). The difference on the perspectives, showed as green arrow between $A$ and $A^{\prime}$, is connected to the metric.

The relation between the Absolute Time and the imaginary time perspective could also be expressed using trigonometric and hyperbolic functions, according to the used basis.

$$
\frac{\overline{O A}}{\overline{O I}}=\tan \left(\frac{\tau_{m}}{2}\right)=\frac{1}{n_{c}}=e^{-\varphi_{c}} ; \quad \frac{\overline{O A^{\prime}}}{\overline{O I}}=\cot \left(\frac{\tau_{m}}{2}\right)=n_{c}=e^{\varphi_{c}}
$$

The value of $n_{c}$ identify the massive particle proper beat in the imaginary time from the Absolute Time perspective. As better elaborated in the following section, the difference between the perspectives from the 2 axes could be connected to the curvature, as evaluating the local information from both the local and the global perspective.

$$
\frac{\overline{A A^{\prime}}}{\overline{O I}}=\frac{\overline{Q Q^{\prime}}}{\overline{O I}}=\left(\frac{\overline{O A^{\prime}}}{\overline{O I}}-\frac{\overline{O A}}{\overline{O I}}\right)=\left(n_{c}-\frac{1}{n_{c}}\right)=2 \cot \left(\tau_{m}\right)=2 \sinh \left(\varphi_{c}\right)
$$

\section{Schwarzschild and Minkowski}

To understand how the metric could be interpreted in the proposed model, we should identify, in the geometric description considered, the relevant points $\left(O, I, A, A^{\prime}\right)$ in the relation between a mass $m$ in space and the curvature of spacetime at a distance $i r$.

We could consider the point $I$ as the Center of Mass (CoM) of the given $m$ while $O$ as the point at distance ir from the CoM, where the curvature is evaluated (defined as CoSelf). The segment $O I$ accounts for the perspective at infinity in a flat space of the distance ir between CoM and CoSelf. The segment $O A$ could be related to the visible information of the mass from the point $O$ at CoSelf and defined as the available Self-reference. The Schwarzschild radius $r_{s}$ of the mass $m$ could be mapped to the segment $I A$, as the summarized information of the mass in space, while $A^{\prime}$ becomes the symmetric projection beyond the singularity, as illustrated in Figure 15.

Following the description of the Apollonius circle, the ratio between the Self-reference and the Absolute perspective could be related to the gravitational information perceived at CoSelf given m@CoM, as the local beat of spacetime from the Absolute Time perspective.

$$
\frac{1}{n_{g}}=\frac{n_{r}-2 n_{m}}{n_{r}}=1-\frac{2 n_{m}}{n_{r}}=1-\left(\frac{2 m}{M}\right)\left(\frac{L}{r}\right)=1-\frac{r_{s}}{r}
$$

The different views on the beat from the Absolute Time and the imaginary time (related to the segment $A A^{\prime}$ in the proposed geometry) could then be related to the metric, through the same relation considered for a particle.

$$
g_{s}=-\left(1-\frac{r_{s}}{r}\right) c^{2} d t^{2}+\left(1-\frac{r_{s}}{r}\right)^{-1} d r^{2}=\left(n_{g}-\frac{1}{n_{g}}\right) d(c t)^{2}=d(i c \tau)^{2}
$$


When $r$ goes to infinity, $A A^{\prime}$ reduces to the diameter of the $\mathrm{BH}$, as the irreducible hidden information in ict, as illustrated in Figure 16. From infinity, given a mass compressed in its information in space to its Schwarzschild radius, a point $A$ on its surface is mapped on the opposite side of the $\mathrm{BH}$ in $A^{\prime}$.

In the absence of mass in the considered universe, no additional hidden information should be available, and the model reduces to a Minkowski metric, resulting in $n_{g}=1$ and $g_{s}=0$.

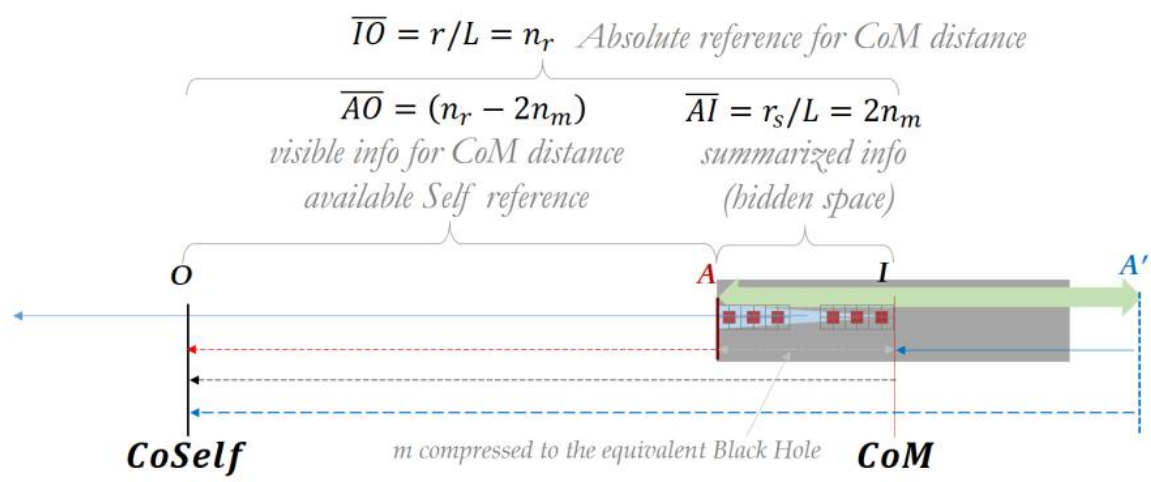

Figure 15. Mapping of the geometric points identified in the model through the Apollonius circle to the analysis of the curvature at a point $O$ at distance $r$ from a mass $m$ located at $I$. The absolute reference on the distance is identified in the segment $I O$, while the visible information is considered the segment $O A$, being $I A$ the equivalent Schwarzschild radius of the mass $m$

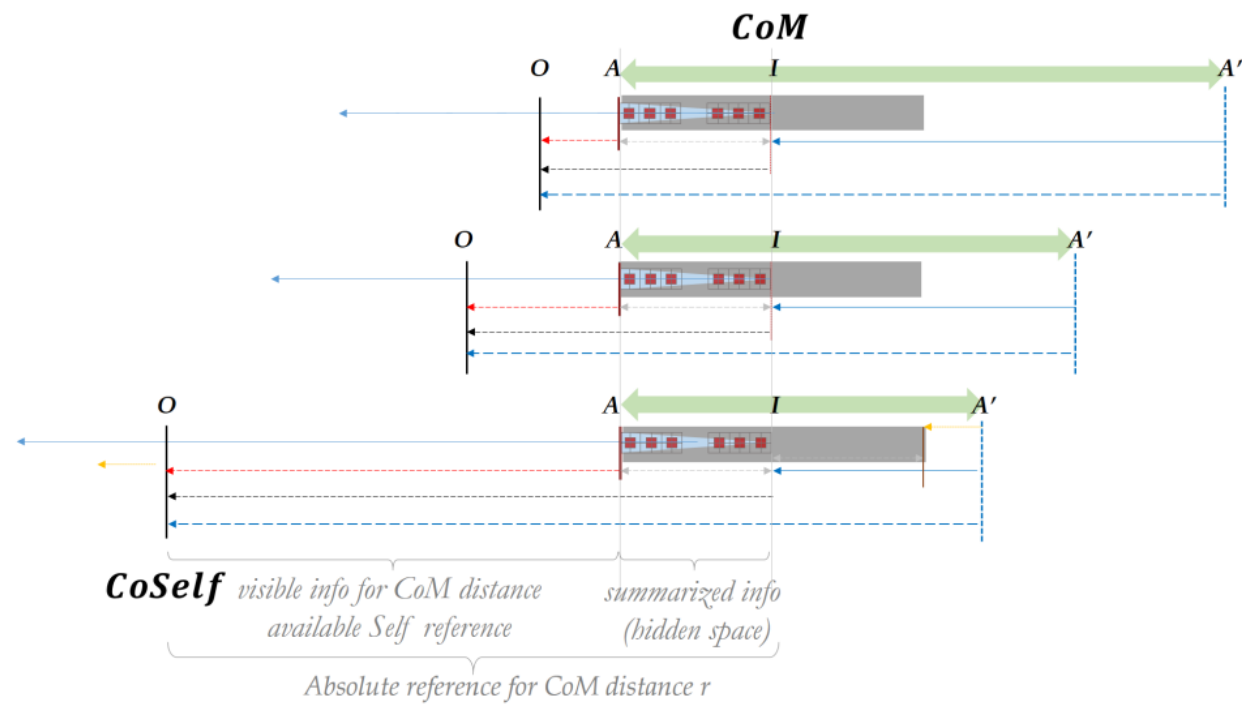

Figure 16. Variations on the distance $r$ of the CoSelf at $O$ from the mass $m$ at CoM (identified by the point $I$ ) and resulting variation in the curvature at $O$ due to the mass $m$. In the limit of the distance $r$ going to infinity, $A A^{\prime}$ reduces to the diameter of the $\mathrm{BH}$, as the total hidden information from the point at infinity on the imaginary time.

We have proposed a geometrical representation of the variables described in the main body of the paper through a mapping on the Riemann sphere and the Apollonius circle. The emergence in ict from $i L$ to infinity (as the Emerging universe perspective) is mapped as inverse on the radius of the parent $\mathrm{BH}$ (BlackHole-universe perspective), from the singularity (at the point $I$ in $i L$ ) to the horizon (at the center of the Apollonius circle). The two perspectives on the same information are related to the beat described in the paper and the difference between them is considered as the basis for metric evaluation, further developed in the last part.

The given description of the relation between the Schwarzschild metric and the variables introduced is not considered as a proper mathematical derivation but tries to offer additional context for the interpretation of spacetime curvature in the proposed geometry. The aim is suggesting the possibility for a more complete framework of analysis for 
spacetime information in terms of a beat in each point in space (derived from the ratio between self and absolute references) and in terms of a metric, intended as a difference between the two perspectives on the considered information.

It is crucial to highlight that the coherence between the information stored in the Absolute Time and the probabilistic space emerging as imaginary time is always maintained in the inversion of perspectives, as long as the references considered leads to a consistent $n_{g} \geq 1$. This implies, in the description of the Schwarzschild metric given, that the point $O$ (at CoSelf where curvature is evaluated) cannot coincide with $A$ (on the horizon of the equivalent $\mathrm{BH}$ related to the mass $m$ ). From $A$ towards the singularity at $I$ (in the $\mathrm{CoM}$ from the outer universe perspective) an inversion of perspectives should be considered. Whenever the CoSelf is inside the $\mathrm{BH}$, it should then be mapped to a vibrating mode in the imaginary time emerging inside the $\mathrm{BH}$.

The singularity at the horizon is considered, in this proposal, as non-reducible, being a discontinuity in the ict axis of the universe containing the BH. The solutions of GR that let the discontinuity disappear (as an artifact of the specific frame of reference) consider the $\mathrm{BH}$ interior as a region of spacetime that still belongs to the same patch and scale with the same references as the outer region. In the suggested interpretation, the inverted perspective should be considered beyond the horizon: the $\mathrm{BH}$ radius should not be addressed as the ict axis of the outer universe through GR but should actually be described as the Absolute Time of the universe inside the $\mathrm{BH}$.

In all other cases, considering the limit of great numbers (ng >> 1), the proposed discrete model should reduce to GR in the continuum regime, but a detailed derivation of Einstein's field equation from the potential in Tk is needed. 


\section{Annex on Expansion}

\section{Evolution as a quantum oscillation}

In the proposed model space has been described as an imaginary distance emerging in each cycle from the causal memories and potential in the Coherent Now. The evolution of space, intended as an expansion in the imaginary time axis as the cycles occur, could be described as a quantum oscillator. We can mathematically describe such behavior considering the minimum mass and the minimum frequency possible at each cycle. We can consider the maximum possible distance in the inner imaginary space at the cycle $k_{\text {in }}$ as the radius of the inner universe expanding beyond the singularity and derive it as the amplitude $A_{\max }$ of the oscillator. We can derive the maximum phase of the inner imaginary universe considering the starting available phase (memory in the $\mathrm{BH}$ radius) and a cumulate phase of causal information, which depends on the number of cycles occurred since the birth of the Absolute Time in the firTk1ss (illustrated in Figure 17).

$$
\begin{gathered}
E_{q o}=\hbar f(n+1 / 2)=\frac{1}{2} \hbar f ; \quad E_{m o}=\frac{1}{2} A^{2} f^{2} m \\
m \rightarrow m_{\text {min }}=\frac{M_{\text {out }}}{n_{b h}}=\frac{M_{\text {in }}}{n_{b h}{ }^{2}} ; \quad f \rightarrow f_{\text {min }}=\frac{1}{t_{\text {uni }}}=\frac{1}{2 k_{\text {in }} T}=\frac{F}{2 k_{\text {in }}} \\
\left(A_{\text {max }} @ k_{\text {in }}\right)^{2}=\frac{\hbar_{\text {in }}}{m_{\text {min }} f_{\text {min }}}=\frac{M_{\text {in }} L_{\text {in }}{ }^{2} F}{\frac{M_{\text {in }}}{n_{\text {bh }}{ }^{2}} \frac{F}{2 k_{\text {in }}}}=2 k_{\text {in }} n_{b h}{ }^{2} L_{\text {in }}{ }^{2}=r_{\text {in }}{ }^{2} \\
r_{\text {in }} @ k_{\text {in }}=L_{\text {in }} e^{\varphi_{\text {in }}}=\sqrt{2 k_{\text {in }} n_{\text {bh }} L_{\text {in }}}=\sqrt{2 k_{\text {in }}} L_{\text {out }} \\
\varphi_{t}=\ln \left(t_{\text {uni }} / T\right)=\ln \left(2 k_{\text {in }}\right) ; \quad \varphi_{\text {bh }}=\ln \left(\frac{m_{b h}{ }^{(k=1)}}{M_{\text {out }}}\right)=\ln \left(n_{b h}\right) \\
\varphi_{\text {in }}=\ln \left(r_{\text {in }} / L_{\text {in }}\right)=\frac{\varphi_{t}}{2}+\varphi_{\text {bh }}
\end{gathered}
$$

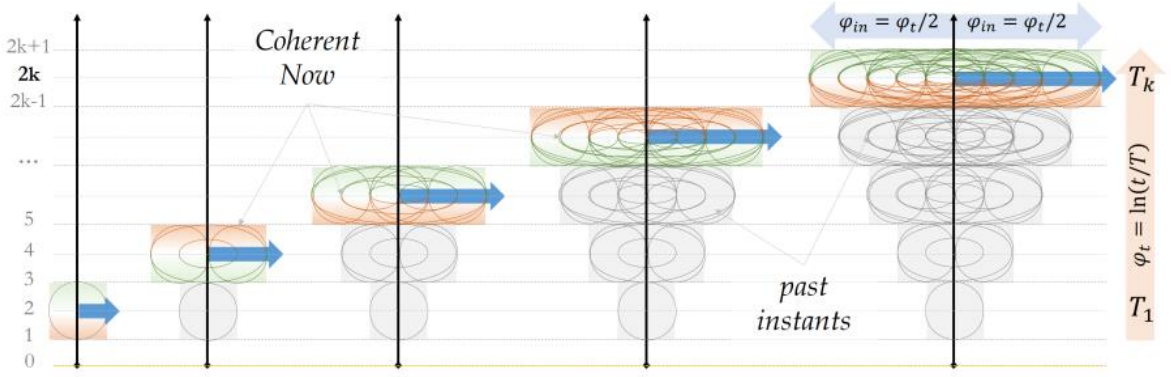

Figure 17. Growth of the inner space ( $\varphi_{\text {in }}$ light blue arrow) as a function of the cumulate phase in the Absolute Time memory ( $\varphi_{t}$ light red arrow). Each current cycle (Coherent Now) is highlighted in shades from red (past) to green (future) while the past instants are illustrated in shades of gray (being non-Real). The Coherent Now includes both the new local possible memory loops and the evolution of the past vibration modes.
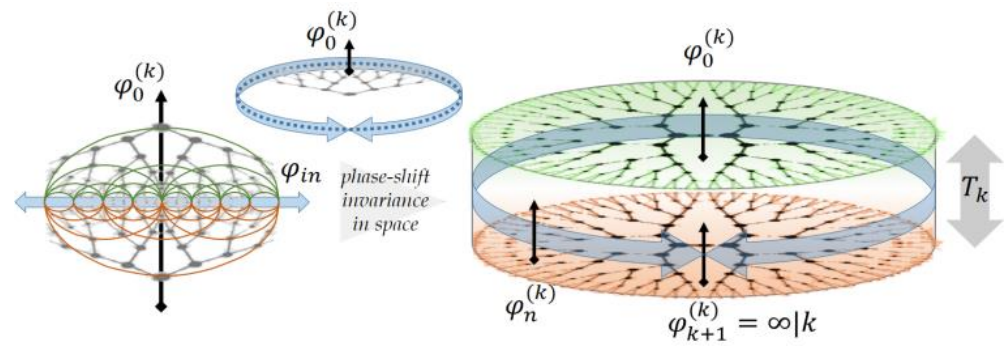

Figure 18. Representation of the inner imaginary space from the CoSelf to infinity as a loop to highlight the phase shift invariance in the imaginary time and the representation as a dual-AdS space from $-T$ and $+T$ perspectives in the Coherent Now. 


\section{Initial conditions and Dark Energy}

We have defined a model of the universe based on momenta and vibration modes stored as phases in a fundamental memory, which evolves on discrete cycles. The starting available memory and phase $\varphi_{b h}$ has been connected to the parent $\mathrm{BH}$ radius at the firs Tk1ss and the inner imaginary space has been described as a quantum oscillator, with an amplitude and equivalent inner imaginary maximum radius that grows following the root of the cosmological time.

It is worth evaluating if and how the vibration modes of the elementary particles' in our universe could be mapped to the hypothetical initial memory, derived from the initial size of the universe after the first cycles. Moreover, considering the size of the universe expressed as a phase $\varphi_{\text {in }}$ at different cycles within the cosmological time, we could try to understand the equivalent growth in mass of the parent $\mathrm{BH}$ containing our universe, intended in the model as a perceived additional Dark Energy that further expands the possibilities in the inner imaginary spatial distance.

$$
\begin{gathered}
m_{b h}{ }^{(k)}=m_{b h}{ }^{(k=1)}+\Delta_{b h}{ }^{(k)} M_{\text {out }} \\
\Delta_{b h}{ }^{(k)}=\sum_{n=1}^{k} \delta_{b h}{ }^{(n)}=\left(\frac{m_{b h}^{(k)}-n_{b h} M_{\text {out }}}{M_{\text {out }}}\right) \\
\varphi_{\Delta}{ }^{(k)}=\ln \left(1+\Delta_{b h}{ }^{(k)} / n_{\text {bh }}\right) \\
\varphi_{\text {in }}=\ln \left(r_{\text {in }} / L_{\text {in }}\right)=\varphi_{b h}{ }^{(k)}+\frac{\varphi_{t}}{2}=\varphi_{\text {bh }}+\varphi_{\Delta}{ }^{(k)}+\frac{\varphi_{t}}{2}
\end{gathered}
$$

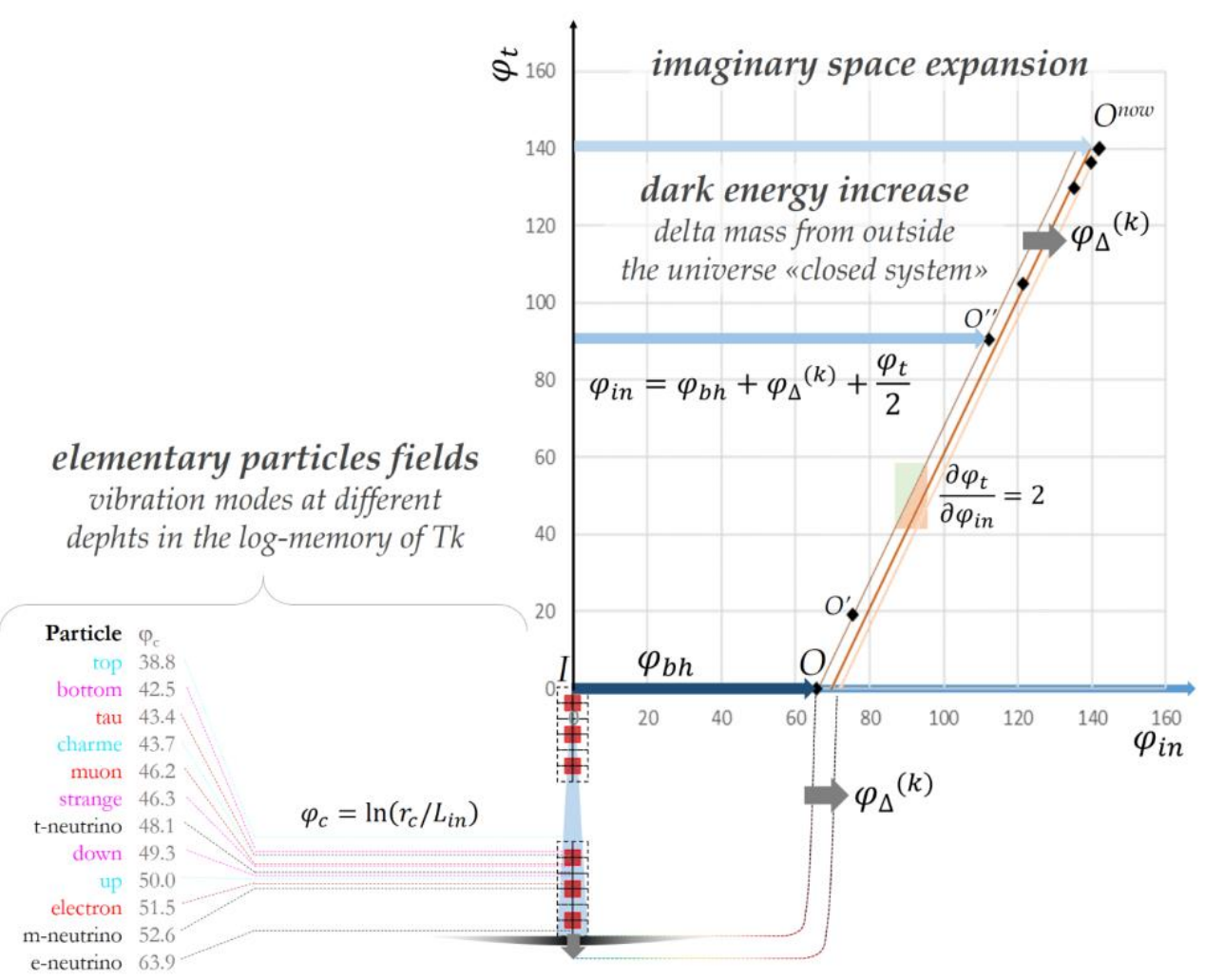

Figure 19. Representation of the elementary particles in the $\mathrm{BH}$ initial memory $\varphi_{b h}$ and of the expanding imaginary space $\varphi_{\text {in }}$ due to the cumulative phase $\varphi_{t}$ (related to the evolution cycles) and the extra phase $\varphi_{\Delta}$ (connected to the mass being absorbed in the parent $\mathrm{BH}$ ). The point $I$ represents the singularity and the point $O$ the most possible distant point at each cycle. 